\title{
Inevitable evolution: back to The Origin and beyond the 20th Century paradigm of contingent evolution by historical natural selection
}

\author{
LARS WiTTING \\ Greenland Institute of Natural Resources, Box 570, DK-3900 Nuuk, Greenland
}

\begin{abstract}
Since neo-Darwinism arose from the work of Darwin and Mendel evolution by natural selection has been seen as contingent and historical being defined by an a posteriori selection process with no a priori laws that explain why evolution on Earth has taken the direction of the major evolutionary trends and transitions instead of any other direction. Recently, however, major life-history trends and transitions have been explained as inevitable because of a deterministic selection that unfolds from the energetic state of the organism and the density-dependent competitive interactions that arise from self-replication in limited environments. I describe differences and similarities between the historical and deterministic selection processes, illustrate concepts using life-history models on large body masses and limited reproductive rates, review life-history evolution with a wider focus on major evolutionary transitions, and propose that biotic evolution is driven by a universal natural selection where the long-term evolution of fitness-related traits is determined mainly by deterministic selection, while contingency is important predominately for neutral traits. Given suitable environmental conditions, it is shown that selection by energetic state and densitydependent competitive interactions unfolds to higher level selection for life-history transitions from simple asexually reproducing self-replicators to large bodied organisms with senescence and sexual reproduction between males and females, and in some cases, to the fully evolved eusocial colony with thousands of offspring workers. This defines an evolutionary arrow of time for open thermodynamic systems with a constant inflow of energy, predicting similar routes for long-term evolution on similar planets.
\end{abstract}

Keywords: Life-history, historical selection, deterministic selection, competitive interactions, density dependence, evolutionary transitions, arrow of time.

\section{Contents}

1 Introduction 2

1.1 Contingent evolution . . . . . . . . . 2

1.2 Inevitable evolution . . . . . . . . 3

1.3 The extent of determinism . . . . . . 6

2 Structure of natural selection $\quad 6$

2.1 Historical selection . . . . . . . . . . 7

2.2 Deterministic selection . . . . . . . 7

2.3 From historical to deterministic selection 8

\section{Historical life-history theory $\quad 9$}

3.1 Density-dependent exploitative competition ............... 9

3.2 Evolution of exploitation . . . . . . . . 10

3.3 Reproduction and body mass . . . . . . 10

3.4 Long-term predictions . . . . . . . . . 11

4 Deterministic life-history theory 12

4.1 Density-dependent interactive competition 12

4.2 Evolution of interactive competition . . 13

4.3 Population limitation . . . . . . . . . . 14

4.4 Reproduction and body mass ... . . . . 14

4.5 Population dynamics . . . . . . . . . . . 16

4.6 Evolution of historical constraints . . . . 17

5 Other life-history traits $\quad 18$

5.1 Multicellular reproducers . . . . . . 18

5.2 Pre-reproductive period and interactive behaviour ............. . . 19

5.3 Metabolic rate . . . . . . . . . . . 19

5.4 Body mass allometries . . . . . . . . . 20

5.5 Senescence and soma . . . . . . . . . . . . 22

5.6 Sexual reproduction . . . . . . . . . . 23

5.7 Eusocial colonies . . . . . . . . . . . . . . 25

5.8 Sessile organisms . . . . . . . . . . 26 


\section{Major life-history transitions}

27

6.1 Models of historical selection . . . . . . 27

6.2 Models of deterministic selection . . . . 28

\section{Evolutionary arrow of time}

7.1 A historic drift . . . . . . . . .

7.2 A deterministic arrow ........ 31

\section{Conclusions}

\section{References}

\section{A Appendix}

\section{Introduction}

The evolution of large organisms with organised phenotypes on Earth was inevitable if natural selection is a deterministic force that applies universally to all life. But the natural selection that was introduced by Darwin (1859), and developed mathematically through the population genetic synthesis (Fisher 1930; Wright 1931; Haldane 1932) and classical life-history theory (reviewed by Charlesworth 1980, 1994; Roff 1992, 2002; Stearns 1992; Charnov 1993; Bulmer 1994; Stearns and Hoekstra 2000), is deeply contingent being a historical and a posteriori selection process with no a priori laws that determine general directions of biotic evolution.

For phenotypic characters that are closely linked to fitness I argue that we need a new paradigm of inevitable evolution based on a universal natural selection that unfolds inevitably and a priori from deterministic laws of self-replication, encompasses historical processes, and defines general directions of biotic evolution. A proposed model of selection by energetic state and density-dependent competitive interactions (Witting 1995, 1997, 2000a,b, 2002a,b,c, 2003a, 2007) illustrates that the evolutionary unfolding of life-history organisation in species on Earth can be explained as arising from first principles of self-replication, predicting that large-scale evolution will follow similar routes on similar planets.

The proposed process does not deny a role for contingency in evolution by natural selection, it finds only that the traditional view is incomplete and that contingency plays a more restricted role than previously assumed. While neutral traits depend on history, opportunity and chance, I argue that fitness-related characters evolve by a deterministic natural selection that unfolds inevitably from self-replication in limited environments.

\subsection{Contingent evolution}

The evolutionary unfolding of biological diversity on Earth can be summarised by major evolutionary trends and transitions. The origin of the first self-replicating molecules initialised a natural selection process where some replicators evolved toward large organisms with high metabolic rates, long pre-reproductive periods, and organised behavioural interactions. Associated with this increase there were transitions from an asomatic, non-senescing, haploid, and asexually reproducing organism to a somatic, senescing, diploid, and sexually reproducing organism with male and female individuals. In some sexually reproducing lineages there were additional transitions into higher level reproducing units of co-operative breeding and eusocial colonies.

A universal theory of evolution by deterministic natural selection would explain the evolutionary history of trends and transitions as arising from first principles of natural selection, just as Newton explained planetary motions from first principles of gravity. But the evolutionary theory of natural selection was constructed the other way around, backwards, by letting natural selection be contingent and defined by evolutionary history. Instead of providing a mathematical theory that explained observed biotic patterns from first principles of natural selection, Darwin proposed that existing species be understood from their evolutionary history of common origin, their heritable variation, and a struggle among individuals for existence.

The approach of defining natural selection from evolutionary history has since become a well-established tradition in evolutionary biology. For example Gould's (2002) odyssey on contingency argues that the evolution of biological organisation cannot in principle be predicted forwards even with full knowledge of antecedent conditions, but can only be understood backwards after time's actual unfolding. This hypothesis forms the basis of historical ecology that analyses the evolutionary diversity of the phylogeny to explain the natural selection process from the phylogenetic constraints of that diversity (e.g. Holder 1983; Brooks and McLennan 1991; McKitrick 1993; Brown 1994; Harvey et al. 1996; Larson and Losos 1996; Martins 1996). It also dominates Dawkins (2004) attempt to understand natural selection through a reverse journey of evolution from present-day humans to the microbial life four billion years ago. Evolutionary theory on major evolutionary transitions (Buss 1987; Maynard Smith and Szathmáry 1995; Michod 1999), sees higher level transitions as evolutionary possibilities given lower level transitions with 'no reason to regard the unique transitions 
as the inevitable result of some general law' (Maynard Smith and Szathmáry 1995, p. 3). 'With the onset of full-blown evolutionism and Darwin's outlook based on branching phylogenies, the very notion that biology might harbour ahistorical universal laws other than "chance and necessity" has become simple nonsense' (Kauffman 1993, p. 5).

The tradition of historicism and contingency has nonetheless been criticised by the adaptationist research program that insists that the process of natural selection must be studied forwards to explain evolutionary history, instead of explaining process from history (e.g. Emlen et al. 1991; Reeve and Sherman 1993, 2001; Brown 1994; Vermeij 1996). But until recently the framework of adaptation has been unable to untangle from the bonds of contingent thinking. Instead of using selection by first principles, explicit models on adaptation have maintained their roots deeply embedded in the historical tradition in the sense that they have diluted evolutionary explanations through circular arguments where the evolutionary history is explained from a natural selection that in turn is explained from the evolutionary history. This is, for example, the case for classical life-history theory (reviewed by Charlesworth 1980, 1994; Roff 1992, 2002; Stearns 1992; Charnov 1993; Bulmer 1994; Stearns and Hoekstra 2000) where the natural selection models that explain the evolution of a phenotypic character of a species are constructed from phenotypic constraints that are soft in the sense that they have evolved in that species during its phylogenetic history. Being a theory that emerged and developed from the mathematical framework of the population genetic synthesis, classical selection theory has always explained phenotypic evolution from selection processes that arise from the genetic architecture, the physiology, or other soft intrinsic constraints that have evolved during the phylogenetic history of the organism.

It is important to understand that it is the evolutionary history of the soft constraints that is the essential component that gives explanatory power to the selection models of classical life-history theory. This is pronounced in selection models that define fitness by the intrinsic Malthusian parameter with the relative fitnesses of variants being constant and independent of their frequencies in the population. These models have, for example, explained the evolution of large body masses from a proportional relationship between body mass and reproductive rate (McLaren 1966; Schoener 1969; Case 1979; Roff 1981, 1986; Lundberg and Persson 1993), and the evolution of limited reproduction from a trade-off between current reproduc- tion and the future survival of the offspring or the reproducing adults (Lack 1947; Schaffer 1983; Godfray et al. 1991; Charlesworth 1994). More elaborate selection models based on frequency-dependent selection have also used soft intrinsic constraints to explain lifehistory evolution. Examples include sex ratio theory where the sex ratio is explained from the ploidy level of the genome that is involved in sexual reproduction (Fisher 1930), and the suite of models that have aimed to explain the evolution of sexual reproduction from genetic recombination (see Section V.6).

The soft constraints in traditional life-history models are often confirmed by observations from populations of existing organisms. But more essentially in relation to their function as explanations for phenotypic evolution, it is important to note that the constraints have not themselves been explained by natural selection. The essential selection pressures are therefore contingent in the sense that they emerge from unknown mechanisms, are being observed a posteriori and, thus, support the 20th Century paradigm that long-term evolution is an unpredictable historical process with no universal determinism to explain why evolution on Earth has taken the direction of the major life-history transitions instead of any other direction. This consensus finds that 'evolution in its original sense of an unfolding or development, analogous to the development of an individual animal, is misleading' (Salthe 1989, p. 134), that natural selection is a 'strictly a posteriori process' that is not 'controlled by any law' (Mayr 1988, pp. 20,105), a process where 'there is no reason why evolution by natural selection should lead to an increase in complexity' (Maynard Smith and Szathmáry 1995, p. 4).

\subsection{Inevitable evolution}

Recently Kauffman (1993, 1995), Witting (1997, 2002b, 2003a), Conway Morris (2003) and Dawkins (2004) have argued that the hypothesis of evolutionary contingency has gone too far. Where Kauffman (1993, 1995) argues that biological order can arise from spontaneous self-organisation in evolving biological systems at the edge of chaos, Conway Morris (2003) and Dawkins (2004) find that the widespread occurrence of convergent evolution, where phenotypic traits like eyes have evolved independently several times on Earth, indicates that there are deterministic-like selection pressures in wide ranges of organisms. In the absence of an explicit theory Dawkins (2004) is cautious and relatively vague on the importance of this determinism, while Conway Morris (2003) argues that physical, chemical and physiological constraints limit the trait-space for alternative 
solutions to a degree where a second rerun of evolution would result in something rather close to man.

Evidence for convergent evolution among diverse organisms on Earth is presented by Conway Morris (2003), mainly for morphological traits but also for life-history traits like body mass, brain size and eusocial reproduction. The hypothesis of inevitable evolution is also statistically supported by parallel evolutionary trajectories in repeated experiments on fastreplicating life forms. Parallel evolution of gene expression (Cooper et al. 2003) and global protein profiles (Pelosi et al. 2006) has been observed in experiments with Escherichia coli, and parallel adaptation to a fluctuating glucose-galactose environment has been observed in experiments with yeasts (Segre et al. 2006). A third not yet applied method for the empirical detection of inevitable evolution is the observation of Earthlike life forms on other planets.

Evolutionary convergence among diverse taxa is clearly empirically supported for some traits. But there is always noise associated with evolutionary data and the observed patterns are not always convincing to everybody. Even Cope's rule (Cope 1871, 1887), which represents one of the best documented evolutionary trends with convergence towards increased body mass in a wide variety of taxa, has long been argued to represent evidence for contingent evolution by neutral evolutionary drift (Stanley 1973; Gould 1988). At a first glance it is probably fair to say that many patterns of large-scale evolution on Earth can be argued to be consistent with both contingent evolution by historical selection and inevitable evolution by deterministic selection. Detailed theoretical analyses of the proposed mechanisms of natural selection are therefore needed in order to determine not only if there are empirical patterns that can differentiate one hypothesis from the other, but also if there is firm theoretical support for the hypotheses.

Throughout this paper I argue that the interspecific life-history patterns that are expected from many of the classical life-history models are inconsistent with empirical patterns, that most of the classical models are evolutionarily unstable in their phenotypic assumptions, and that they therefore offer no firm theoretical support for the hypothesis of contingent evolution. When analysed in detail it seems that contingent evolution by historical natural selection is only vaguely supported both empirically and theoretically.

In parallel with my critique of contingency I argue that evolution can indeed be predicted forwardly, if not necessarily for individual species in practice, then, at least in terms of overall evolutionary trends, transi- tions, and patterns. Most of my arguments are based on Malthusian Relativity (Witting 1995, 1997, 2000a,b, $2002 a, b, c, 2003 a, 2007$ ), a mathematical selection theory with density- and frequency-dependent relativity in the relative fitnesses that are defined by the Malthusian parameter. This theory finds that major life-history trends and transitions evolve by a deterministic selection pressure that arises from the energetic state of the organism and the density-dependent competitive interactions between interacting individuals in natural populations. It is only if we can construct a theory of deterministic natural selection, where the essential selection forces unfold automatically from first principles of selfreplication, that we can obtain a truly predictable natural selection that will explain a priori why organisms with organised phenotypes evolve in the long run. Such a theory should explain phenotypic traits not only from the proximate selection forces that operate on them, but also why the observed selection forces have come into existence in the first place.

Given self-replication and organisms at a sufficiently high level of organisation, it is rather obvious that several morphological traits must evolve inevitably from the deterministic selection pressure of the physicalchemical environment. Mobility, for example, is beneficial for heterotroph organisms that depend on small resource items that are patchily distributed, and eyes are beneficial for the behaviour of mobile organisms in illuminated environments. But instead of focussing on traits that evolve by physical-chemical selection I deal primarily with the ultimate selection causes for the evolution of life-history characters like large body masses, high metabolic rates, long pre-reproductive periods, body mass allometries, senescence, males, sexual reproduction and eusocial colonies. Because all lifehistory characters are closely linked to fitness, if anything, it is their evolution that best reveals whether evolution by natural selection is primarily contingent or inevitable.

Nearly all life-history traits that distinguish large and organised organisms from the initial self-replicator at the origin of natural selection are closely linked by energetic trade-offs to the intrinsic Malthusian parameter, which defines fitness for the frequency-independent selection of the historical life-history models with constant relative fitnesses. First expectations for long-term evolution by natural selection are therefore the absence of these traits. Generally speaking, it seems that these characters can evolve and continue to exist only if there is a persistent frequency-dependent selection that will outbalance their frequency-independent cost.

In the search for a frequency-dependent selection that 
will explain large and organised organisms as a universal principle of evolution I return to the origin of natural selection theory. Here Darwin (1859) paid special attention not only to the Malthusian principle, that 'a struggle for existence inevitably follows from the high rate at which all organic beings tend to increase' (p. 63), but he also noted that 'the struggle almost invariably will be most severe between the individuals of the same species' (p. 75). But Darwin never pursued this insight to show how selection by density-dependent interactive competition among individuals in natural populations selects for the biological diversity on Earth. Nor was this principle taken seriously as a necessary and nearly sufficient mechanism for the evolution of large and organised life forms before it was used by Witting (1995, 1997, 2000a,b, 2002a,b,c, 2003a, 2007) in his theory of Malthusian Relativity to develop a deterministic natural selection for the evolutionary unfolding of major life-history trends and transitions in mobile organisms. Being one long argument for a return towards an ecological basis for evolutionary biology, this theory aims to explain overall patterns of life-history evolution from ecological components extrinsic to organisms, in particular from the density-dependent competitive interactions among individuals in natural populations.

Selection models with competitive interactions among individual organisms have been described under the game theoretical concepts of evolutionarily stable strategies (Maynard Smith and Price 1973; Maynard Smith 1982; see also Vincent and Brown 1988; VegaRedondo 1996; Dugatkin and Reeve 1998; Hofbauer and Sigmund 1998), and continuously stable strategies (Eshel and Motro 1981; Eshel 1983; see also Taylor 1989; Christiansen 1991; Metz et al. 1992; Metz et al. 1996a; Dieckmann 1997; Eshel et al. 1997; Geritz et al. 1997, 1998; Kisdi 1999). Realising that the genetic structure evolves in response to natural selection on longer-time scales the continuously stable strategies of genetic-free models can be used as predictors of long-term evolutionary attractors (Eshel and Feldman 1984, 2001; Liberman 1988; Matessi and Eshel 1992; Hammerstein 1996; Matessi and Di Pasquale 1996; Eshel et al. 1997; Eshel et al. 1998).

Game theories of evolutionarily and continuously stable strategies were originally developed for an evolutionary understanding of behavioural interactions given the implicit assumption that the level of interactive behaviour is constant and density independent. More recently there has been increasing focus on the evolutionary importance of including density dependence in the level of interactive behaviour into analytical models (e.g. Abrams and Matsuda 1994; Mylius and Diekmann
1995; Metz et al. 1996b; Witting 1997, 2002b, 2003a; Heino et al. 1998; Gyllenberg and Parvinen 2001; Dercole et al. 2002; Dieckmann and Metz 2006). Interest in extending the hypothesis of selection by densitydependent competitive interactions into a general lifehistory theory, however, has remained surprisingly low (Day and Taylor 1996), with the work of Witting (1995, 1997, 2000a,b, 2002a,b,c, 2003a, 2007) being an exception that not only incorporates density dependence in the number of competitive interactions into a general selection framework for the evolution of major lifehistory characters, but also appears to be the only framework that aims for a deterministic natural selection that excludes soft historical constraints from the assumptions of selection models.

Selection by density-dependent competitive interactions is one of two major selection principles in Malthusian Relativity. This principle recognises that selfreplication generates population growth that results in increased densities of self-replicators generating increased interactive competition that selects for an increase in interactive quality. Increased interactive quality can, among other things, be obtained by life-history characters like body mass, metabolic rate, interactive behaviour, senescence, sexual reproduction, and higher level reproducing units including eusocial colonies (Witting 1997, 2002b, 2003a). The second principle is based on the energetic state of the organism, and recognises that self-replicators at higher energetic states tend to be selected over self-replicators at lower states because organisms at higher states have more energy to allocate into fitness-enhancing traits like reproduction, survival or interactive quality. Organisms at higher energetic states have potentials for populations with higher densities and higher levels of interactive competition. This potential implies that major life-history trends and transitions that are selected for by interactive competition can unfold as a function of an evolutionary increase in energetic state (Witting 2003a).

Given physical-chemical conditions that are favourable for life, I propose that not only is selection by energetic state and density-dependent competitive interactions a sufficient mechanism for the evolution of large organisms with organised phenotypes, but it is also a necessary mechanism. We will find that the majority of the life-history traits that distinguish higher organisms from simple self-replicators can be explained by this selection mechanism independently of soft constraints. This, however, does not imply that the selection pressure on a life-history cannot be influenced by that life-history. It implies only that the trait components under natural selection that do 
so can, at least in principle, be forwardly predicted from first principles of self-replication in limited environments. Nor do I suggest that the life-history traits under consideration are unaffected by other selection forces than those explicitly considered. I suggest only that most of these other selection forces are secondary forces that are insufficient and not necessary when it comes to the evolutionary existence of these life-histories. Indeed, as we shall see, even though selection by exploitative competition is likely to affect most of the traits that I deal with, I find that selection by exploitative competition alone cannot explain the evolutionary existence of these traits, at least not unless we assume some unwarranted form of contingency that is imposed by unexplained soft phenotypic constraints.

\subsection{The extent of determinism}

It is only characters that are linked to fitness that may evolve inevitably by deterministic natural selection, while neutral characters and trait components with no relation to fitness necessarily will be contingent being the result of history, opportunity and chance. The latter characters, however, will not evolve by natural selection but by mutation, drift and diffusion. It is only if the historically determined neutral characters subsequently induce natural selection on other traits that we can have contingent evolution by historical natural selection on the latter traits (see Section II.3).

A first suggestion for the extent of evolutionary historicism and determinism is given by the natural selection models within the two concepts that are valid both theoretically and empirically. While it is often impossible to determine if a selection model is strictly valid in the sense that it gives the true explanation for the evolution of a phenotypic character, it is often possible to determine if a model is invalid in the sense that it cannot in itself represent the true explanation.

Throughout this paper I argue that many of the historical selection models are invalid theoretical explanations of contingent evolution for the simple reason that the model predictions are evolutionarily unstable in the assumed soft phenotypic constraints. In addition to this theoretical argument we note that historical selection models with constant relative fitnesses are falsified empirically if the relevant relative fitnesses in natural populations are frequency-dependent, and that the suggested deterministic selection models with frequencydependent selection are falsified if the relevant relative fitnesses turn out to be constant. For the life-history characters that are analysed herein it seems that the relevant relative fitnesses nearly always are frequencydependent.

The domain of determinism in natural selection is likely to extend beyond my analysis which is restricted to major life-history traits. As already noted, deterministic predictions are often straightforward for morphological traits like the eye that evolve by physicalchemical selection pressures. To understand the potential limit to determinism it is essential to distinguish a trait, as defined by its main function, from the design solution through which this function is expressed. Almost any design problem, including the design of characters under natural selection, has several possible solutions. Thus, while selection for the main function of a character may arise deterministically from self-replication, the explicit choice for one of the many potential design solutions is, in many cases, presumably the result of phenotypic history, opportunity, and chance. Where deterministic natural selection may explain the evolution of a trait (like an eye), or variation in the values of a trait (like responses of the maximal reproductive rate to variation in the rate of extrinsic mortality), the chosen details of the design solution (such as the relative distribution of the different tissues in the eye) is likely to be left to the realm of contingency.

Here I analyse and compare the predictions of available models under the two hypotheses of contingent evolution by historical selection and inevitable evolution by deterministic selection. Historical and deterministic life-history models for the evolution of body mass and reproductive rate are described in detail. The extension of principles into a general theory of life-history evolution is given by brief reviews on the evolution of reproducers, multicellular organisms, a prolonged pre-reproductive period, behavioural interactions, high metabolic rates, body mass allometries, senescence, sexual reproduction and eusocial colonies. The overall frameworks are then considered in relation to major evolutionary transitions, and a timeasymmetry of the deterministic selection mechanism is described to define an evolutionary arrow of time for open thermodynamic systems with a constant influx of energy. First, I examine the structure of natural selection theory to determine when there is mathematical support for the hypothesis of contingent evolution by historical selection and the hypothesis of inevitable evolution by deterministic selection.

\section{Structure of natural selection}

To describe the structure of natural selection let the phenotype of interest $\mathbf{P}$ be characterised by a set of 
traits $\mathbf{P}=\left\{q_{1}, q_{2}, \ldots, q_{k}\right\}$. Let $\mathbf{P}^{\mathbf{c}}$ be the complete parameter space of all possible phenotypes given the set of traits, and $\mathbf{P}^{* \mathbf{i}}$ a parameter space that is fundamental, also referred to as initial, in the sense that it represents the complete phenotypic space of the initial self-replicators that can define the origin of natural selection. Let $\mathbf{P}^{i}$ be a specific parameterisation, like a continuously stable strategy (CSS, denoted $\mathbf{P}^{* *}$ ), or a specific parameterisation $\mathbf{P}^{* i}$ in $\mathbf{P}^{* i}$.

\subsection{Historical selection}

The classical life-history theory that is reviewed by Charlesworth (1980, 1994), Roff (1992, 2002), Stearns (1992), Charnov (1993), Bulmer (1994) and Stearns and Hoekstra (2000) is based mainly on models of historical natural selection. These models typically split the phenotype $\mathbf{P}$ into two sub-sets $\mathbf{P}=\mathbf{P}_{e} \cup \mathbf{P}_{c}$ : the lifehistory traits $\mathbf{P}_{e}$ that are explained by a natural selection that is defined by the historical constraints $\mathbf{P}_{c}$. These constraints are soft in the sense that they are phenotypic components that evolve over time, and they include physiological trade-offs or genetic constraints that define relationships between traits. Alternatively the constraints may be phenotypic traits that take specific values.

With a single CSS that is also a global attractor the causal structure of a historical selection model can be described as

$$
\mathbf{P}_{e \mid \mathbf{P}_{c}^{i}}^{* *}=F\left(\mathbf{P}_{c}^{i}, \mathbf{P}_{e}^{\mathbf{c}}, E\right)
$$

where the phenotypic state of the CSS $\left(\mathbf{P}_{e \mid \mathbf{P}_{c}^{i}}^{* *}\right)$ is given by a selection function $(F)$ that is defined mainly by the soft constraints $\left(\mathbf{P}_{c}^{i}\right)$, but typically also by an extrinsic environment $(E)$ and the complete parameter space of the traits to be explained $\left(\mathbf{P}_{e}^{\mathbf{c}}\right)$. For a specific historical parameterisation $\mathbf{P}_{c}^{i}$, there may be multiple CSSs, where the CSS to evolve $\mathbf{P}_{e \mid \mathbf{P}_{c}^{i}, \mathbf{P}_{e}^{\mathbf{i}}}^{* *}=F\left(\mathbf{P}_{c}^{i}, \mathbf{P}_{e}^{\mathbf{i}}, E\right)$ depends on the initial conditions $\mathbf{P}_{e}^{\mathbf{i}}$ of the phenotypic traits to be explained, with $\mathbf{P}_{e}^{\mathbf{i}}$ defining the trait space where evolution proceeds towards the $\mathbf{P}_{e \mid \mathbf{P}_{c}^{i}, \mathbf{P}_{e}^{i}}^{* *}$ attractor.

As the soft constraints that generate the predictions of historical selection models are given by assumption and/or observation instead of being explained by natural selection, the scope of the predictions is restricted in the sense that it does not ultimately explain why the phenotype has evolved. To explain fully the long-term evolution of a trait it is not sufficient to deduce the trait from observed existing selection pressures, the proximate selection pressures need instead to be explained from first principles of natural selection. Long-term evolution by historical selection is therefore contingent and inherently unpredictable because the natural selection process is defined by soft constraints that have evolved by unknown mechanisms.

\subsection{Deterministic selection}

A selection is truly deterministic only if it is deducible from the self-replication process of the initial self-replicator that defines the origin of natural selection. This requirement imposes strong limitations to the intrinsic constraints that can be incorporated into models of deterministic natural selection. Only hard constraints, that cannot be explained by natural selection because they are given by laws that lie outside the domain of evolutionary biology, can be used (Witting 1997, 2002b, 2003a). These constraints include the traits of the initial self-replicator at the origin of natural selection, the self-replicating process itself, and the physical-chemical environment. Given these axiomatic conditions it is especially the ecological constraints that arise from self-replication in limited environments that may form the basis for life-history models of deterministic selection.

A challenging task for models of deterministic selection is the construction of the most fundamental models where the relationships among the different traits reflect only hard constraints that cannot be altered by natural selection. A first attempt for such models was developed for mobile organisms by Witting (1997, $2002 b, 2003 a$ ), who assumes that there are no hard constraints between life-history traits unless we have a non-biological law that clearly explains why the different traits should be linked to one another, for example, through energetic trade-offs. Although this assumption of trait independence might not reflect the true constraints of natural selection, it is likely to be a best first principle because it prevents traits from being evolutionarily explained from soft constraints that are evolutionarily modifiable. These limitations imply a selection function with the following structure

$$
\mathbf{P}^{* *}=F\left(\mathbf{P}^{* \mathbf{i}}, E\right)
$$

where the phenotypic state of the CSS $\left(\mathbf{P}^{* *}\right)$ is given by a selection function $(F)$ that arises from self-replication in the limited environment $(E)$ given an initial phenotype within the parameter space of simple selfreplicators $\left(\mathbf{P}^{* \mathbf{i}}\right)$.

Like historical models deterministic models may have multiple CSSs, with evolution towards a specific attractor being dependent upon the initial conditions of the 
traits to be explained $\left[\mathbf{P}_{\mid \mathbf{P}^{\mathbf{i}}}^{* *}=F\left(\mathbf{P}^{\mathbf{i}}, E\right)\right.$, with $\mathbf{P}^{\mathbf{i}}$ defining the trait space where evolution proceeds toward the $\mathbf{P}_{\mid \mathbf{P}^{\mathbf{i}}}^{* *}$ attractor]. In such cases there might be only a single CSS that has an initial trait space $\left(\mathbf{P}^{\mathbf{i}}\right)$ that includes the phenotypic space of simple self-replicators $\left(\mathbf{P}^{* \mathbf{i}}\right)$. All the attractors, however, are part of the deterministic model because they are based on the same deterministic selection model, with the complete phenotypic state of each of the attractors being maintained by a natural selection with no soft constraints and with transitions between the attractors being given by initial conditions.

Models of deterministic selection may be nested to explain larger sets of traits. The following model

$$
\begin{aligned}
\mathbf{P}_{1}^{* *} & =F_{1}\left(\mathbf{P}_{1}^{* \mathrm{i}}, E\right) \\
\mathbf{P}_{2 \mid \mathbf{P}_{1}^{* *}}^{* *} & =F_{2}\left[F_{1}\left(\mathbf{P}_{1}^{* \mathbf{i}}, E\right), \mathbf{P}_{2}^{* \mathbf{i}}, E\right]
\end{aligned}
$$

for example, will deduce a CSS for the combined phenotype $\left(\mathbf{P}=\mathbf{P}_{1} \cup \mathbf{P}_{2}\right)$ from the initial state $\left(\mathbf{P}^{* \mathbf{i}}=\right.$ $\left.\mathbf{P}_{1}^{* \mathbf{i}} \cup \mathbf{P}_{2}^{* \mathbf{i}}\right)$ and an environment $(E)$. By exchanging $F_{1}\left(\mathbf{P}_{1}^{* \mathbf{i}}, E\right)$ with $\mathbf{P}_{1}^{* *}$ in eqn 4 , we obtain a selection structure

$$
\mathbf{P}_{2 \mid \mathbf{P}_{1}^{* *}}^{* *}=F_{2}\left(\mathbf{P}_{1}^{* *}, \mathbf{P}_{2}^{* \mathbf{i}}, E\right)
$$

that has a close resemblance with historical selection (eqn 1). Nevertheless, the joint selection function of eqns 3 to 5 represents a deterministic model because the soft constraint $\left(\mathbf{P}_{1}^{* *}\right)$ that is essential for the higher level selection function (eqn 4) is already explained by a deterministic selection at a lower phenotypic level (eqn 3). This implies that a deterministic selection model on a trait is not necessarily mutually exclusive to a historical selection model on the same trait: If $\mathbf{P}_{1}^{* *}$ of eqn 5 is identical to $\mathbf{P}_{c}^{i}$ of eqn 1, then the joint selection function of eqn 5 can include the historical selection model as a sub-model of the overall deterministic model. This is possible because the soft constraint $\left(\mathbf{P}_{1}^{* *}\right)$ of the deterministic model is a predicted CSS, while for the historical model the constraint $\left(\mathbf{P}_{c}^{i}\right)$ is given by assumption instead of being explained by natural selection. Normally, however, proposed models of historical and deterministic selection on the same traits are not fully integrable, although they may be combined to gain insights into the evolution of the soft constraints in the historical models (see Section IV.6).

As the hard constraints that are incorporated into deterministic selection models are either physicalchemical constraints or constraints that arise from the origin of natural selection, i.e. self-replication, it follows that deterministic models may ultimately explain why a given phenotype has evolved by natural selection. Evo- lution by deterministic selection models is thus longterm predictable and inevitable if the physical-chemical conditions allow evolution to proceed in the predicted directions.

While deterministic models may be used to explain general life-history patterns of long-term evolution, they are restricted to the degree that they cannot explain evolutionary components that depend on history and initial conditions. This implies that not all traits may be explained by deterministic selection. Neutral traits are the obvious exception. They will always be unpredictable with their current values being given exclusively by their evolutionary history. These traits, however, are generally not interesting in terms of models of natural selection, unless they have some influence on the evolutionary attractors of other traits. Note also that life-history traits that evolve by deterministic selection in the long run might be explained in more detail by historical selection if their current evolutionary state is limited by soft constraints.

\subsection{From historical to deterministic selection}

Historical selection models are truly historical only if the soft constraints that define the selection pressure of the models are selectively neutral. If not, then, given sufficient time in a stable extrinsic environment the constraints should evolve, typically towards an evolutionary attractor. The soft constraints of a historical selection may therefore take a long-term transition where they either vanish, because they are relaxed by natural selection, or merge into the set of traits that are explained by first principles leaving the set of unexplained historical traits empty. In either case, with no unexplained traits, the historical model has evolved into a model of deterministic selection giving theoretical support to the hypothesis of inevitable evolution at the cost of the original hypothesis of contingent evolution by historical constraints.

The soft constraints in models of historical selection are normally treated as being unaffected by the selection pressures of the models even though the implicit model structure often defines selection on them. When, for example, a constraint is the lack of genetic variation in a discrete trait, a single mutation can induce an evolutionary transition from the constraint $\left(\mathbf{P}_{c}\right)$ to an evolutionary equilibrium $\left(\mathbf{P}_{c}^{* *}\right)$. In such cases it is often easy to transform a historical model $\left[\mathbf{P}_{e \mid \mathbf{P}_{c}^{i}}^{* *}=F\left(\mathbf{P}_{c}^{i}, \mathbf{P}_{e}^{\mathbf{c}}, E\right)\right]$ into a deterministic counterpart $\left[\mathbf{P}^{* *}=F\left(\mathbf{P}^{* \mathbf{i}}, E\right)\right]$ to examine if a phenotypic transition is expected in the long run.

For historical models with implicit selection on soft 
constraints, the equilibria of the historical and deterministic version of the same model may coincide, confirming that the original historical hypothesis can straightforwardly be converted into a deterministic hypothesis for the long-term evolution of the phenotype predicted by the historical model. But more often the phenotypic equilibrium of the two model versions will differ from one another. For most of the models examined herein, the CSS of the deterministic version of the historical model falls within the phenotypic space of the initial self-replicator. The historical model will then not only fail as theoretical support for the original hypothesis of contingent evolution, but it will also fail as a predictor for the long-term evolution of a derived phenotype predicting instead the long-term evolutionary maintenance of the initial self-replicator.

If a model of contingent evolution by historical selection fails because it predicts the inevitable long-term evolutionary maintenance of the initial self-replicator, it may still have merit on an immediate time scale where it may provide insights into processes of life-history optimisation. Many models of historical selection have been constructed so that both the assumed soft constraints and the predicted traits coincide with the traits in the species to which the model is applied. In such cases, the historical models can use the observed proximate selection pressure to explain the phenotype of an existing organism to the degree, and only to the degree, that it can be determined whether the phenotype is currently in evolutionary equilibrium or not. In this way a historical model may be used to understand how phenotypic traits are adjusted and optimised in relation to one another given an observed selection pressure, while at the same time the model does not explain why the phenotype or the observed selection pressure has evolved. The models are then not really models of life-history evolution, but instead a practical tool that can be used to analyse the evolutionary status of the life-history.

\section{$3 \quad$ Historical life-history theory}

In the search for a life-history theory of long-term evolution let me first consider the historical selection models from classical life-history theory. The analysis will start from the population dynamic ecology associated with the historical selection models, continue through the ecological implications of selection, and end with life-history evolution.

\subsection{Density-dependent exploitative competi- tion}

The influence of density dependence on life-history evolution has often been neglected. Within more than a thousand pages of reviews of life-history theory (Stearns 1992; Stearns and Hoekstra 2000; Roff 2002), densitydependent selection is mentioned briefly only on five pages. Selection has instead been defined by the Malthusian parameter, given either as the per generation growth rate $\lambda$ or as the instantaneous rate of increase $r=\ln \lambda$. Here it has often been assumed that the relative fitnesses of variants are constant and independent of their frequencies in the population, which in effect reduces fitness to the intrinsic (genetically coded) Malthusian parameter.

Since exponential and geometrical population growth is unrealistic over time scales of long-term evolution, the assumption of constant relative fitnesses is best seen as reflecting density regulation by exploitative competition under the assumption of no exploitative interactions among variants (interactive competition might also in principle affect variants symmetrically causing the relative fitnesses to be constant, but such cases are very rare if occurring at all among natural organisms and are thus not considered herein). With nonoverlapping generations and density dependence acting on fecundity, this implies that the population equation

$$
N_{t+1}=\lambda_{t} N_{t}
$$

has the following form of density dependence

$$
\lambda_{t}=p \hat{R} f\left(N_{t}\right)
$$

that applies not only to the population average but also to all the genetic variants in the population $\left[N_{t}\right.$ is population density in generation $t, \hat{R}$ the intrinsic (denoted by hat) component to lifetime reproduction $(R)$, $p$ the probability of survival to the reproductive age, and $f(N)$ the density regulation function that declines monotonically with $N]$.

True density-dependent selection by exploitative competition, where the relative fitnesses among variants depend on density, may occur when the density dependence on the demographic parameters of a variant depends not only on density, but also on the demographic parameters of that variant $[\lambda=$ $p(\hat{p}, N) R(\hat{R}, N)$, where $\hat{p}$ is the intrinsic survival probability]. For a more detailed coverage of densitydependent selection under exploitative competition see Roughgarden (1971), Charlesworth (1971, 1994), Kimura (1978), Ginzburg (1983), Boyce (1984) and Mylius and Diekmann (1995). 
Density dependence by exploitative competition only is unrealistic for nearly all, if not all, natural populations for the simple reason that individuals interact. It could though, at least in principle, be sufficient for models of life-history evolution should interactive competition be irrelevant in relation to fitness and natural selection, which would be the case if interactive competition affects variants symmetrically.

Independent of exploitative and interactive competition, in itself, all density regulation implies a population limitation that is intrinsically determined in the sense that the carrying capacity (abundance at population dynamic equilibrium, denoted by $*$, and defined by $\lambda^{*}=1$ ) is a secondary effect of the intrinsic components of the demographic parameters:

$$
N^{*}=f^{-1}(1 / p \hat{R})
$$

with the inverse density regulation function $f^{-1}$ increasing monotonically with $p \hat{R}$. Under traditional limitation, any factor that causes a change in reproduction or survival is a limiting factor that affects the equilibrium population density (see review by Sinclair 1989).

\subsection{Evolution of exploitation}

With fitness being defined by the intrinsic Malthusian parameter Fisher (1930) introduced the Fundamental Theorem of Natural Selection: 'The rate of increase in fitness of any organism at any time is equal to its genetic variance in fitness at that time' (Fisher, 1999, p. 35). There soon grew the misinterpretation that the theorem described an overall increase in the average Malthusian parameter of a population (e.g. Wright 1930, 1955; Li 1955; Crow and Kimura 1956; Kempthorne 1957; Kimura 1958, 1965; Kojima and Kelleher 1960; Ewens 1969). But as pointed out by Price (1972) and noted by Fisher (1930) himself, owing to density dependence the increase in fitness produces only a partial increase in the Malthusian parameter: 'The balance left over when from the rate of increase in the mean value of $m$ produced by Natural Selection, is deducted the rate of decrease due to deterioration in environment, results not in an increase in the average value of $m$, for this average value cannot greatly exceed zero, but principally in a steady increase in population' (Fisher, 1999, p. 42). This insight that the carrying capacity can be a fitness measure under natural selection optimisation was later stressed by Nicholson (1960) and developed mathematically by Roughgarden (1971), Charlesworth (1971, 1994), Kimura (1978) and Boyce (1984).

The predicted continued increase in carrying capacity by natural selection generates a nature that is unbal- anced with population densities that increase to levels where resources are highly overexploited. This prediction is in great contrast to the fact that many primary producers are almost unaffected by the presence of herbivores, especially in terrestrial ecosystems where they eat less than $10 \%$ of the edible plant biomass (e.g. Wiegert and Owen 1971; Ricklefs 1990; Hairston and Hairston 1993).

Instead of focussing on the absence of intraspecific interactive competition in selection theory, Hairston et al. (1960) proposed and Hairston and Hairston (1993) elaborated the hypothesis that resources are rather unexploited because predators limit herbivores. Dependent upon parameter values this argument may hold for the case with no evolutionary changes. But on larger time scales with selection by exploitative competition, the hypothesis of predator-limited herbivores requires a Red Queen scenario (Van Valen 1973) where the evolutionary rates in all species outbalance one another so that there is no overall evolution. The evolutionary rates among species could in principle be balanced in this way in some instances, but there is no reason to expect that a balance is guaranteed. In the absence of a balance the density of at least one of the involved species will increase in an uncontrolled manner that is not normally observed for natural populations.

Wynne-Edwards (1962, 1986, 1993) argued instead that individual selection is flawed owing to its inability to explain a nature in balance. He suggested that group selection sets upper limits to reproduction because over-exploitation causes the extinction of groups having any higher rate of reproduction. But the required conditions for group selection where individuals retain from reproduction for the benefit of the group are so restricted that group selection generally cannot explain a nature in balance (Williams 1966).

\subsection{Reproduction and body mass}

In relation to life-history evolution I have chosen limited reproductive rates and non-negligible body masses as illustrative examples. Both of these traits are central to our understanding of life-history evolution, among the most widespread life-histories in natural organisms, and have a long history in the scientific literature on life-history evolution.

With natural selection that favours the most successful replicators in a population, we expect that selection should allocate energy into reproduction at the cost of investment in mass, generating an unbalanced nature with steadily increasing equilibrium densities. Large body masses, limited reproductive potentials and lim- 
ited population densities were therefore seen as an evolutionary paradox causing Lack (1947) to propose that limited reproduction evolves because the future survival $(p)$ of offspring and/or parents is negatively related to the number of offspring produced by the parents [e.g. $p \propto e^{-c_{r} R}$, with $R$ being lifetime reproduction and $c_{r}$ a constant that defines the trade-off, see Charnov and Krebs (1974), Schaffer (1983), Godfray et al. (1991) and Roff (2002) for details]. Later McLaren (1966), Roff (1981, 1986), Gerritsen (1984) and others (Stearns and Koella 1986; Lundberg and Persson 1993) proposed that large body masses evolve from a proportional relationship $(R \propto w)$ between body mass $(w)$ and lifetime reproduction, with the additional constraint that survival declines with mass (e.g. $p \propto e^{-c_{w} w}$, with $c_{w}$ defining the trade-off). This implies constant relative fitnesses for both models and population dynamics that is either density independent or density-dependent by exploitative competition. The associated selection profiles $[r=\ln \lambda=\ln (p R)]$ on body mass $\left(r \propto \ln w-c_{w} w\right)$ and reproductive rate $\left(r \propto \ln R-c_{r} R\right)$ have selection gradients $\left(\partial r / \partial w=1 / w-c_{w}\right.$ and $\left.\partial r / \partial R=1 / R-c_{r}\right)$ that select for CSSs with a non-negligible body mass $\left(w^{* *}=1 / c_{w}\right)$ and a limited reproductive potential $\left(R^{* *}=1 / c_{r}\right)$.

\subsection{Long-term predictions}

While the classical selection models appear to solve the paradox of limited reproduction, large body masses, and a nature in balance, the solution does not hold on the evolutionary scale. This is because the predictions are evolutionarily unstable in the sense that they depend on the soft constraints of the models. For Lack's (1947) model on limited reproduction the trade-off between future survival and the current reproductive effort is likely to reflect a hard energetic constraint, but the absolute scaling of the trade-off, as described by the parameter $c_{r}$, is soft and evolving by natural selection.

Given Lack's (1947) hypothesis the selection gradient on the trade-off parameter $\left(c_{r}\right)$ is negative $\left(\partial r / \partial c_{r}=\right.$ $-R$ ). This implies a long-term transition to a phenotype with a continuous increase in reproductive potential $\left(c_{r} \rightarrow 0 \Rightarrow R^{* *}=1 / c_{r} \rightarrow \infty\right)$. With persistent directional selection on the soft constraint of the tradeoff, Lack's (1947) hypothesis is not really a model of contingent evolution because this would require that the trade-off parameter was selectively neutral. In the deterministic version, Lack's (1947) model selects for infinite reproduction offering no solution to the paradox that a limited reproductive potential has evolved in many species. To solve this paradox we need instead to explain from first principles either the evolutionary scaling of the trade-off between current reproduction and future survival or the evolution of limited reproduction independently of the trade-off.

Where the soft constraint in Lack's (1947) model on limited reproduction is a modifiable trade-off, the essential constraint in historical selection models on body mass is the proportional relation between the reproductive rate and body mass. While this relationship is observed in several species (Wooton 1979; Ridely and Thompson 1979; Peters 1983; Reiss 1989), the mechanism behind it is unclear and there is no reason to expect that the relationship is a hard constraint that cannot evolve by natural selection. Likely best candidates for a hard and evolutionarily fixed relationship between body mass and reproductive rate are constraints based on energetic trade-offs. To identify such a trade-off let the energetic state $(\epsilon)$ of an organism be defined by the net attainable resource that is available for lifetime reproduction and/or an increased body mass in the reproducing individual. The energy available for lifetime reproduction is then $\epsilon_{R}=\epsilon-(1-\rho) w$, where $(1-\rho) w$ is the energy that an individual allocates to its own growth, and $0<\rho \leq 1$ the energetic fraction of the adult body mass that parents allocate into each offspring. Thus, with the total parental investment in the body mass of an offspring being $\rho w$, there is an inverse relationship between body mass and lifetime reproduction

$$
R=[\epsilon-(1-\rho) w] / \rho w .
$$

With $\epsilon-(1-\rho) w$ amounts of energy available for reproduction $[\epsilon-(1-\rho) w] / \rho w$ offspring can be produced giving natural selection the choice of allocating resources to body mass or reproductive rate.

The inverse relationship of eqn 9 implies a fitness profile

$$
r=\ln (p[\epsilon-(1-\rho) w] / \rho w)
$$

that induces persistent selection for a negligible adult body mass and a negligible parental investment in each offspring. While the traditional models on body mass evolution may use an observed relationship between body mass and reproductive rate as an immediate measure to determine if the body mass is in evolutionary equilibrium, these models do not offer a solution to the paradox that non-negligible body masses and large parental investments per offspring have evolved in many species. To solve this paradox we need to explain from first principles either the evolution of the proportional relationship between body mass and reproductive rate, or the evolution of non-negligible body masses independently of the observed relationship between body mass 
and reproductive rate.

The limitation to the classical selection framework is not only theoretical. There is also an important empirical inconsistency if we assume that the models apply not only on the immediate time scale but also on the longer time scale of evolutionary change. On the latter scale, the assumption of constant relative fitnesses implies that the models operate under the Fundamental Theorem of Natural Selection where the intrinsic Malthusian parameter can only increase with life-history evolution, generating an increase in the population dynamic growth potential or carrying capacity or both. Thus, with larger body masses and lower reproductive rates being the more evolved traits that generally evolve from smaller body masses and higher reproductive rates, our first expectation from constant relative fitnesses is that organisms with larger body masses and lower reproductive rates have higher intrinsic Malthusian parameters than organisms with smaller body masses and higher reproductive rates. Not only is this prediction against intuition, but it is also contrary to evidence, where the intrinsic Malthusian parameter generally is positively related to the reproductive rate, it scales to the negative 1 /4-power of body mass (Fenchel 1974), and abundance scales to the negative 3/4-power of body mass (Damuth 1981, 1987).

The Fundamental Theorem of Natural Selection does not apply to the frequency-dependent selection of density-dependent competitive interactions (Witting $2000 a, 2002 c$ ), where in fact the intrinsic Malthusian parameter may decline with natural selection explaining its negative correlation with body mass (Section V.4). Thus, given that non-negligible body masses and limited reproductive rates evolve by natural selection it can be concluded that their evolution is driven by frequency-dependent selection. The classical selection models with constant relative fitnesses are therefore failing on the scale of long-term evolution, while their framework may be applied at the immediate time scale where there are no changes in the environment or in the frequencies of variants in the population and, thus, by definition the relative fitnesses are constant.

\section{Deterministic life-history theory}

Let me now examine if deterministic selection by the energetic state of the organism and the density-dependent competitive interactions that arise from the population growth of self-replication can explain the long-term evolution of non-negligible body masses and limited reproductive rates.

\subsection{Density-dependent interactive competi- tion}

For classical life-history models with constant relative fitnesses the effect of competition on an individual organism is indirect reflecting the response of the individual to the overall level of resources that is left over given the total resource exploitation of the population. With interactive competition added the effect of competition becomes direct and asymmetrical. This implies that the competitive effect on an individual is a function not only of the interactive (competitive) quality of that individual, but also of the interactive quality of the other individuals in the population, and of the density-dependent frequency of competitive encounters among individuals.

In order to incorporate interactive competition into selection models we need to define density dependence more explicitly than in traditional life-history models. Using $f(N)=N^{-\gamma}$ as an approximation of density dependence in the surroundings of the population dynamic equilibrium, it follows that the density dependence of the energetic state of the organisms is linear on a logarithmic scale

$$
\ln \epsilon=\ln \hat{\epsilon}-\gamma_{\alpha} \ln N-\mu \iota
$$

with

$$
\iota=\gamma_{\iota} \ln N
$$

and the intrinsic component to the energetic state being defined here by the energetic state of an unexploited resource $(\hat{\epsilon})$, and the overall density regulation $(\gamma=$ $\gamma_{\alpha}+\mu \gamma_{\iota}$ ) reflecting the two components of exploitative $\left(\gamma_{\alpha}\right)$ and interactive $(\mu \iota)$ competition, with $\mu$ being the cost (payoff) per competitive interaction, $\iota$ the level of interactive competition that reflects the number of competitive encounters per interacting unit (individual) per unit time, and $\gamma_{\iota}$ the density dependence in the number of competitive interactions.

Relative to exploitative competition, interactive competition is asymmetrical in the sense that it biases fitness in favour of the interactively superior individuals that can dominate the inferior individuals during competitive encounters and obtain a disproportionately large fraction of the resources. To describe this let body mass, both as adult body mass $(w)$ and as the relative mass of juveniles to adults $(\rho)$, reflect interactive quality; other things being equal, it is often easier to monopolise a resource if you are larger than your opponents. Let $w$ and $\rho$ be the geometric average of the two traits in the population, let $w_{i}$ and $\rho_{i}$ be the traits of the $i$ th variant, $\mu$ the average cost associated with a competitive encounter for the average variant in the 
population, and $\mu_{i}=\mu+\Delta \mu_{i}$ the average cost for the $i$ th variant. The difference in cost between the $i$ th and the average variant is then

$$
\begin{aligned}
\Delta \mu_{i} & =\mu_{i}-\mu \\
& =\psi \ln \left(w / w_{i}\right)+\psi_{\rho} \ln \left(\rho / \rho_{i}\right)
\end{aligned}
$$

where $\psi$ and $\psi_{\rho}$ are the within-population slopes between the two traits of interactive quality and the cost of interactive competition on logarithmic scale. Hence, with density-dependent competitive interactions added the fitness profile of exploitative competition (eqn 10) extends into

$$
\begin{aligned}
r_{i}^{*}= & \ln \left(p\left[\epsilon^{*}-\left(1-\rho_{i}\right) w_{i}\right] / \rho_{i} w_{i}\right) \\
& +\psi \iota^{*} \ln \left(w_{i} / w\right)+\psi_{\rho} \iota^{*} \ln \left(\rho_{i} / \rho\right)
\end{aligned}
$$

which describes the fitness of the $i$ th variant as a function of the life-history of that variant, the average lifehistory in the population, and the density-dependent level of interactive competition in the population (the energetic state of an individual at population dynamic equilibrium is $\epsilon^{*}=\hat{\epsilon} N^{*-\gamma}$ ).

\subsection{Evolution of interactive competition}

The evolution of the level of interactive competition at population dynamic equilibrium $\left(\iota^{*}\right)$ is one of the most central and influential predictions from selection by density-dependent competitive interactions. This is because it is the level of interactive competition that is the crucial ecological component that determines whether there is selection for an increase or decrease in interactive traits like body mass and parental investment per offspring.

If the level of interactive competition is sufficiently low there is selection for a decline in interactive quality. This is because interactive quality implies a fitness cost in the sense that quality requires energy that otherwise could be used to enhance the rate of self-replication, and with no interactions it does not pay to invest in interactive quality. If instead the level of interactive competition is sufficiently high there is selection for an increase in interactive quality because the interactively superior individuals can dominate the inferior individuals and obtain so much extra resource that they can have a higher realised replication than the interactively inferior individuals even though intrinsic replication is fastest for the latter individuals.

There is usually a stable evolutionary equilibrium to the level of interactive competition at population dynamic equilibrium. This is because there is a negative feedback loop between the level of interactive competition at population dynamic equilibrium and the average interactive quality in the population. This loop exists because the level of interactive competition is a function of population density (eqn 12), with the population density at population dynamic equilibrium being positively related to the demographic parameters (eqn 8), while these parameters are negatively related to interactive quality (eqn 9). Hence, if interactive quality is low, the population density and the level of interactive competition is high and selection is likely to favour an increase in interactive quality. If instead interactive quality is high, the level of interactive competition is low and selection is likely to favour a decline in interactive quality. In between there is an evolutionary equilibrium where the interactive quality of the organisms is balanced against the level of interactive competition at population dynamic equilibrium.

Most of the interactive traits considered in this study and by Witting (1997, 2002b, 2003a) have only a limited ability to control the level of interactive competition in the population. This is either because the traits can take only a limited range of values and/or because their influence on lifetime reproduction is relatively limited, allowing only for some evolutionary adjustment to the population level of interactive competition. Body mass that covers 21 orders of magnitude, from $10^{-13} \mathrm{~g}$ for microbes to $10^{8} \mathrm{~g}$ for whales, however, is the exception to this rule. Owing to its huge quantitative range and inverse relation to lifetime reproduction (eqn 9), body mass has the potential almost fully to adjust the level of interactive competition from zero to infinity. Selection on body mass is therefore expected to act as an evolutionary buffer that will adjust the population level of interactive competition to an equilibrium where there no longer is selection for an increase or decline in body mass. The level of interactive competition at this equilibrium will then determine the selection pressure on the other traits of interactive quality. And if evolutionary changes in the other traits follow, we expect some further evolutionary buffering by the body mass in order to maintain the level of interactive competition relatively constant while energy is selected from the body mass to the other interactive traits, or the other way around.

Describing this process mathematically, from eqn 14, the selection gradient on body mass is

$$
\left.\frac{\partial r_{i}^{*}}{\partial w_{i}}\right|_{w_{i}=w}=\left[\psi \iota^{*}-1-\frac{(1-\rho) w}{\epsilon^{*}-(1-\rho) w}\right] \frac{1}{w}
$$

with $\left.\right|_{w_{i}=w}$ denoting that the partial derivative that is taken across the gradient of variants in the popu- 
lation is to be taken at the limit of the average body mass in the population. From the $p R^{*}=1$ constraint of the population dynamic equilibrium, which is given by eqn 14 at the limit of the average variant, we have $p\left[\epsilon^{*}-(1-\rho) w\right] / \rho w=1$ and thus $\epsilon^{*}=w(1-\rho+\rho / p)$. Inserting this $\epsilon^{*}$ in eqn 15 , and with the evolutionary adjustment of body mass operating through an evolutionary adjustment of the level of interactive competition, from $\partial r_{i}^{*} /\left.\partial w_{i}\right|_{w_{i}=w}=0$ we find that the equilibrium level of interactive competition that generates a body mass in evolutionary equilibrium is

$$
\iota^{* *}=\left[1+\frac{p(1-\rho)}{\rho}\right] \frac{1}{\psi}
$$

or simply

$$
\iota^{* *}=1 / \psi
$$

when $\rho=1$. The level of interactive competition at evolutionary equilibrium is thus independent of the body mass and energetic state of the organism.

More generally, there are four evolutionary states to the level of interactive competition in the population (Witting 1997, 2002b, 2003a). The results above assume an organism with a non-negligible energetic state. If instead the organism is a low-energy self-replicator, the energetic state may be so low that even with the smallest of all possible body masses the individuals cannot generate population growth, and consequently interactive competition, at a level that matches that of the evolutionary equilibrium. Hence, initial selfreplicators at a sufficiently low energetic state will experience almost an absence of interactive competition $\left(\iota^{* i} \approx 0\right.$; with $* i$ denoting the downward constrained equilibrium of the initial self-replicator). The equilibrium of eqn 16 furthermore assumes an energetic state in evolutionary equilibrium. If instead, as expected for organisms on resources that are freely exploited, there is selection for an increase in energetic state, we can expect an evolutionary steady-state with a somewhat increased level of interactive competition $\left(\iota^{* s} \approx 7 / 3 \psi\right.$ for $\rho=1$ and foraging in two spatial dimensions; with $* s$ denoting evolutionary steady-state) and an exponential increase in energetic state and body mass. If there are special constraints that set an upper limit on body mass that is lower than expected from the energetic state of the organism, then the level of interactive competition can evolve to almost infinite levels $\left(\iota^{* c} \approx \infty\right.$; with $* c$ denoting upward constrained equilibrium). As described in the following, these four levels of interactive competition not only limit the population density, but also play a crucial role for life-history evolution in general.

\subsection{Population limitation}

In Section III we saw that the traditional view of population limitation is an equilibrium density that is determined by the demographic parameters (eqn 8), while the demographic parameters are determined independently by natural selection. But with the historical selection models failing at the evolutionary scale, longterm limitation may be determined by deterministic selection by density-dependent competitive interactions.

Given selection by density-dependent competitive interactions it follows that the equilibrium population density is defined by the evolutionarily determined level of interactive competition. Here it is the ecological constraints of the equilibrium level of interactive competition $\left(\iota^{* *}\right)$ and the density dependence of interactive competition $\left(\iota=\gamma_{\iota} \ln N\right)$ that set the equilibrium population density to

$$
N^{* *}=e^{\iota^{* *} / \gamma_{\iota}}
$$

given the density dependence of eqns 11 and 12 . Hence, there is no general selection towards an evolutionary increase in carrying capacity, and contrary to traditional population regulation (eqn 8), the intrinsic Malthusian parameter and its two demographic components of survival $(p)$ and reproduction $(\hat{R})$ are absent from the limiting factors that determine the equilibrium density. In fact, the mechanism of population limitation is turned up side down. It is now the intrinsic Malthusian parameter and the product between its two demographic components $p$ and $\hat{R}$ that is determined by an equilibrium population density that is adjusted to the evolutionary equilibrium level of interactive competition in the population:

$$
p \hat{R}=1 / f\left(N^{* *}\right)
$$

with $N^{* *}$ being given by eqn 18 . There is no longer a theoretical paradox associated with the observed balance of nature. On the contrary there is indeed a balance where the intrinsic rates of survival and reproduction are evolutionarily adjusted to match the equilibrium density and the extrinsic imposed mortality $\left[\hat{p} \hat{R}=1 / f\left(N^{* *}\right) \check{p}\right.$, with $p=\hat{p} \check{p}$ under the assumption of independent survival owing to intrinsic $(\hat{p})$ and extrinsic $(\check{p})$ causes].

\subsection{Reproduction and body mass}

Interactive competition may result in evolutionary "arms races" where frequency-dependent selection for interactive quality generates a directional evolution where an interactive trait continues to absorb energy 
from the reproductive rate causing the intrinsic Malthusian parameter and the population dynamic equilibrium to decline towards zero and, thus, the population to evolve towards extinction (Simpson 1953; Dawkins and Krebs 1979; Parker 1979, 1983; Haigh and Rose 1980; Maynard Smith 1982; Maynard Smith and Brown 1986; Brown and Maurer 1986; Vermeij 1987; Härdling 1999). The directional evolution of arms races applies only if the level of interactive competition is sufficiently large and density independent (Abrams and Matsuda 1994; Witting 1997, 2003a), but the level of interactive competition is density-dependent in most, if not in all, natural populations.

With density-dependent interactive competition it follows that selection for or against an additional investment in each offspring is determined by the evolutionarily determined level of interactive competition:

$$
\left.\frac{\partial r_{i}^{*}}{\partial \rho_{i}}\right|_{\rho_{i}=\rho}=\left[\psi \iota^{* *}-1+\frac{\rho w}{\epsilon^{*}-(1-\rho) w}\right] \frac{1}{\rho} .
$$

By inserting the energetic state at population dynamic equilibrium $\left[\epsilon^{*}=w(1-\rho+\rho / p)\right]$ and the evolutionary equilibrium level of interactive competition (eqn 16) into eqn 20 we find that there is persistent selection for an increase in offspring investment when $\psi_{\rho} / \psi>1-p$, i.e., when the interactive importance of offspring investment relative to body mass is larger than the cumulated probability of dying before reproduction. Hence, when survival is sufficiently high, parental investment will evolve to a maximum $\left(\rho^{* *}=1\right)$, where parents produce offspring with body masses similar to those of adults. If instead $\psi_{\rho} / \psi<1-p$, which is most likely when the chances of surviving to reproduce are low, parental investment in each offspring may evolve to be negligible, with the relative mass of offspring to adults being $\rho^{* *}=\frac{p}{1-p} \frac{\psi_{\rho}}{\psi-\psi_{\rho}}$.

Owing to the ability of the body mass to adjust the level of interactive competition in the population, a full description of the selection gradient on body mass needs to incorporate the functional dependence of interactive competition on body mass at population dynamic equilibrium. With $\rho^{* *}=1$, from eqns 9 , 11 , and 12 the population dynamic equilibrium is defined by $p R^{*}=p \hat{\epsilon} N^{-\gamma} / w=1$. This implies that the body mass dependence of the equilibrium density is $N^{*}=(p \hat{\epsilon} / w)^{1 / \gamma}$, and that the body mass dependence for the level of interactive competition at population dynamic equilibrium is $\iota^{*}=\left(\gamma_{\iota} / \gamma\right) \ln (p \hat{\epsilon} / w)$. By inserting this into eqn 14 we find that the fitness profile that incorporates density and body mass dependence in the level of interactive competition is

$$
r_{i}^{*}=\ln \left(p \epsilon^{*} / w_{i}\right)+\left(\psi \gamma_{\iota} / \gamma\right) \ln (p \hat{\epsilon} / w) \ln \left(w_{i} / w\right) .
$$

Thus, there is a neutrally stable equilibrium to the body mass and reproductive rate (for details see Witting 2003a):

$$
w^{* *}=p \epsilon^{* *}
$$

and

$$
\hat{R}^{* *}=\hat{\epsilon} / w^{* *},
$$

with $\epsilon^{* *}=\hat{\epsilon} N^{* *-\gamma}=\hat{\epsilon} e^{-\iota^{* *} \gamma / \gamma_{\iota}}=\hat{\epsilon} e^{-\gamma / \gamma_{\iota} \psi}$. While it is the density-dependent competitive interactions that select for changes in body mass, it is mainly the energetic state and the level of mortality that determine the equilibrium value for body mass. It is intriguing that the evolutionary equilibrium for the body mass is defined by the density-dependent constraint $\left(p \epsilon^{*} / w=1\right)$ of the evolutionarily determined population dynamic equilibrium. This is because of the evolutionary buffering where the body mass is adjusted to generate a reproductive rate that produces an equilibrium abundance that matches the fixed level of interactive competition at evolutionary equilibrium. If mortality $1-p$ is increased there will be a reallocation of energy from body mass to increased reproduction in order to maintain the level of interactive competition constant. If instead, there is an increase in energetic state $\hat{\epsilon}$, for example, by an increase in resource production, natural selection will allocate the extra available energy into body mass while the level of interactive competition and the reproductive rate will remain the same.

Intermediate body masses and reproductive rates are also expected at the evolutionary steady-state (Witting, $2002 b, 2003 a$ ), where the level of interactive competition is somewhat above the level at evolutionary equilibrium, and there is an exponential evolutionary increase in body mass and energetic state. Hence, where historical selection models by exploitative competition generally predict the long-term evolution of negligible body masses, and "arms races" by density independent competitive interactions predict body masses at the other extreme where populations become extinct, selection by density-dependent competitive interactions strikes a balance where evolution proceeds towards body masses and reproductive rates at intermediate levels.

An intermediate body mass, however, is not always guaranteed by selection by density-dependent competitive interactions (Witting, 2002b, 2003a). Some selfreplicators may have energetic states that are so low that they have populations with almost no interactive 
competition, which implies constant selection for a decline in body mass. They are thus expected to be at a downward constrained equilibrium where the body mass is as small as possible if it is not being maintained by another selection mechanism. And at the other extreme, with special upper constraints on body mass and a sufficiently high energetic state, the level of interactive competition can increase to almost infinite levels, generating constant selection for a body mass increase while the mass remains constant at the upper limit. Such systems generate selection for eusocial colonies (Section V.7), and when these have evolved they may change the mode of interactive competition so that the interactive quality of the colony is determined more by the number of individuals in the colony than by the body mass of each individual.

\subsection{Population dynamics}

The evolutionary equilibrium for selection by densitydependent competitive interactions is an equilibrium not only for life-history evolution but also for population dynamics (Witting 1997, 2000b, 2002a). Traditionally following Malthus (1798), Lotka (1925) and Volterra (1926) population dynamics have been defined independently of evolutionary changes (but see Chitty 1960, 1967; Ginzburg 1980, 1983; Saccheri and Hanski 2006). This implies that unchecked populations increase geometrically by the Malthusian law (Malthus 1798), and that density-regulated populations return monotonically to the population dynamic equilibrium unless large reproductive potentials and strong density dependence in association with age-structure or nonoverlapping generations generate fluctuating or chaotic dynamics (May 1976; May and Oster 1976; Oster 1976; Charlesworth 1980; Nisbet and Gurney 1982). But population cycles in natural populations most often take several generations, and this requires a delayed density dependence that traditionally has been explained by predator-prey interactions in Lotka-Volterra-like equations (see reviews by Norrdahl 1995; Batzli 1996; Berryman 1996; Krebs 1996; Ginzburg and Colyvan 2004). The best documented cycles in insects, however, lack a firm predator-prey interaction (Berryman 1996), and despite extensive research efforts 'there is not a single field experiment to show that predator removal has any impact on cycles of voles or lemmings' (Krebs 1996, p. $13)$.

With selection by density-dependent competitive interactions the population dynamic equilibrium is not only maintaining the population abundance in a balance between population explosion and population ex- tinction; it is also maintaining the life-history in a balance between a continued evolutionary increase in interactive quality and a continued decline towards the initial self-replicator. If density-dependent interactions are removed from the population dynamic selection model it follows that the energetic trade-off between interactive quality and the demographic parameters selects for a life-history collapse towards the initial selfreplicator, while at the same time the intrinsic Malthusian parameter and the carrying capacity would increase as predicted by historical selection models with constant relative fitnesses. And if density dependence is removed altogether, the unchecked population would increase hyper-geometrically owing to the Malthusian law of geometrical increase for fixed demographic parameters and Fisher's Fundamental Theorem of Natural Selection that selects for a geometrical increase in the intrinsic Malthusian parameter (Witting 1997, 2000b).

Given density-dependent competitive interactions and selection on interactive traits like body mass, the population dynamics of density dependence [i.e. $N_{t+1}=$ $N_{t} \hat{\lambda} N_{t}^{-\gamma}$ from eqns 6 and 7 given $f(N)=N^{-\gamma}$ ] evolves into inertia dynamics

$$
\begin{aligned}
\hat{\lambda}_{t} & =\hat{\lambda}_{t-1} N_{t-1}^{\gamma_{q}} e^{\sigma^{2}} \\
N_{t+1} & =N_{t} \hat{\lambda}_{t} N_{t}^{-\gamma}
\end{aligned}
$$

where $\sigma^{2}$ is the additive genetic variance of interactive quality and $\gamma_{q}=\gamma_{\iota} \psi \sigma^{2}$ is an inertia parameter that determines the degree of delayed density dependence caused by natural selection [see Witting (1997, $2000 b, 2002 a$ ) for the evolution of inertia dynamics; and Ginzburg and Taneyhill (1994), Ginzburg (1998), Witting (2003b), and Ginzburg and Colyvan (2004) for dynamic implications].

The expected evolutionary changes in life-history traits will induce an additional layer of density dependence that is delayed by a single generation, and this generates a single-species mechanism for the population cycles that have fascinated ecologists for decades (Fig. 1). Owing to the possibility of a repeated cyclic selection pressure from eqns 24 and 25, for frequently perturbed populations we may expect long-term selection for plastic phenotypes that respond not only to the effects of direct density dependence but also to the delayed density-dependent effects of natural selection. Cyclic dynamics from selection by density-dependent competitive interactions may thus not only reflect direct genetic responses to selection, but also plastic responses like maternal effects (Ginzburg and Taneyhill 1994; Ginzburg 1998; Ginzburg and Colyvan 2004).

It can therefore be concluded that density-dependent 


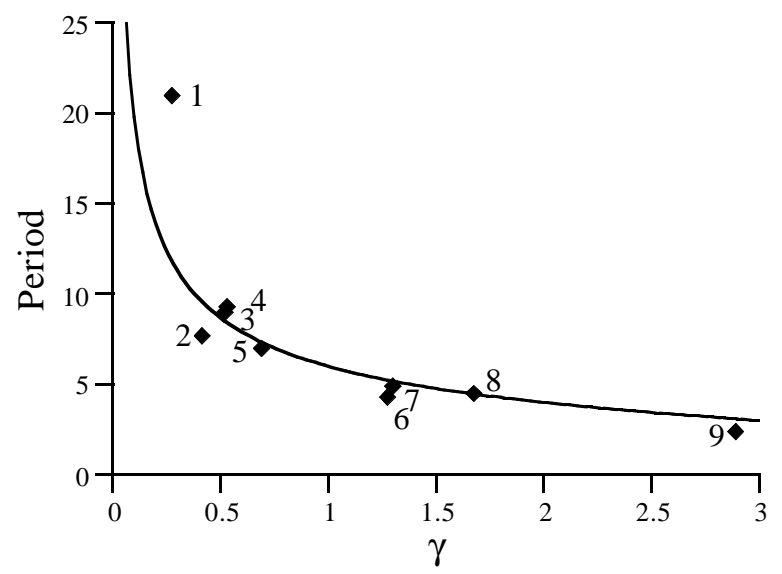

Figure 1: The period of the population dynamic cycle in generations against the strength of density regulation $(\gamma)$ and inertia $\left(\gamma_{q}\right)$ in eqns 24 and 25, assuming neutrally stable cycles, i.e. $\gamma=\gamma_{q}$. The curve is given by eqns 24 and 25, and the numbered points represent the following species: 1 spruce budworm, Choristoneura fumiferana; 2 southern pine beetle, Dendroctonus frontalis; 3 douglas-fir tussock moth, Orgyia pseudotsugata; 4 larch budmoth, Zeiraphera diniana; 5 fall webworm, Hyphantria cunea; 6 nun moth, Lymantria monacha; 7 pine looper moth, Bupalus piniarius; 8 larch cone fly, Strobilomyia sp.; 9 wasp, Vespula sp. From Witting (1997).

competitive interactions provide an explicit mechanism for the Chitty $(1960,1967)$ hypothesis that population cycles are driven by natural selection, a mechanism that has now been shown to induce two-year oscillation in side-blotched lizards, Cuta stansburiana (Sinervo et al. 2000). Population cycles caused by selection by interactive competition also implies cyclic changes in the lifehistory characters of interactive quality (Witting 1997, $2000 b$ ), and such cycles have often been observed in natural populations with cyclic dynamics (e.g. Krebs and Myers 1974; Krebs 1978; Boonstra and Krebs 1979; Stenseth 1982; Stenseth and Ims 1993; Lidicker and Ostfeld 1991; MacColl et al. 2000). As these life-history cycles tend to be out of phase with the effects of density regulation, i.e. with larger individuals occurring at peak densities where smaller individuals are expected by over-exploitation alone, their explanation requires an additional factor like selection by density-dependent competitive interactions.

\subsection{Evolution of historical constraints}

Here I investigate if selection by density-dependent competitive interactions can be combined with the historical selection models to explain the evolution of the soft constraints in the latter models. The underlying hypothesis is that the two approaches represent dual theories that focus on different aspects of the same natural selection, with deterministic selection operating on the evolutionary time scale so that the physiology of individual organisms and the life-history covariance among organisms within populations reflect the constraints of this selection. Then, at the immediate time scale, where fitnesses among variants are constant because the population density is fixed and there are no evolutionary changes, the classical framework with constant relative fitnesses can be applied.

For Lack's (1947) model of limited reproduction this implies that the trade-off between current reproductive effort and future survival is adjusted by natural selection so that the physiological optimum to the reproductive rate coincides with the reproductive rate for the equilibrium of selection by energetic state and densitydependent competitive interactions. Thus, given a lifehistory in evolutionary equilibrium, the reproductive rate $R^{* *}$ is given by selection by density-dependent competitive interactions, and Lack's (1947) model can be applied to determine the evolutionary setting of the physiological trade-off. To see this let the reproductive effort, which was expressed by $R$ in Section III.3, be rewritten as $w R$ to reflect the energy that is allocated into the body mass of the offspring. If this effort is scaled by the constant $R^{* *}$, the tradeoff between intrinsic survival and reproductive rate is $p \propto e^{-c_{r} w R / R^{* *}}$, which implies a selection gradient of $\partial r / \partial R=1 / R-c_{r} w / R^{* *}$. At equilibrium $R=c_{r} w / R^{* *}$ we have that $R=R^{* *}$, and that the trade-off parameter $c_{r}$ depends on body mass $c_{r}=1 / w$. Hence, if the physiology is optimised in accordance with the tradeoff from Lack's (1947) model, it follows that variation in body mass will induce variation in the trade-off between the reproductive effort and future survival. Body mass selection by density-dependent competitive interactions is therefore expected to adjust the trade-off to some equilibrium level $c_{r}^{* *}=1 / w^{* *}$ that reflects the evolutionary determinance of body mass.

Hence, when Lack's (1947) model is integrated with selection by density-dependent competitive interactions, the long-term evolution of limited reproduction is determined relatively independently of Lack's (1947) model. The integration, however, allows us to explain the evolution of the trade-off that originally was given by assumption and seen as the cause for the evolution of limited reproduction. In conclusion we find that the evolutionary causality of Lack's (1947) hypothesis is reversed: according to the original hypothesis it is the Malthusian parameter and its demographic compo- 
nents of survival and reproduction, and thus also the equilibrium population density, that are evolutionarily determined by the trade-off between current reproduction and the future survival of offspring and adults. According to the combined model of Lack's (1947) hypothesis and density-dependent competitive interactions it is instead the Malthusian parameter, its demographic components and the trade-off between them that are evolutionarily determined by a population density that evolves to meet the constraining level of interactive competition at evolutionary equilibrium.

The proportional relationship between the reproductive rate and body mass that selects for a non-negligible body mass in the historical life-history theory is an unexplained observation of within-population life-history covariance. The relationship may therefore be seen as a test for the hypothesis of selection by energetic state and density-dependent competitive interactions. If correct the latter hypothesis should be able to explain the relationship from first principles.

Given that there is no constraint between body mass and the probability of surviving to reproduce, the selection gradient of density-dependent competitive interactions predicts that the intrapopulation variation in life-time reproduction is independent of body mass at the evolutionary equilibrium, and that it is approximately proportional to body mass at the evolutionary steady-state (Witting 1997, 2003a). The often observed proportionality between lifetime reproduction and body mass thus suggests that many natural populations are situated at the evolutionary steady-state with an exponentially increasing energetic state and body mass.

\section{Other life-history traits}

We have now seen that models of historical natural selection on limited reproduction and non-negligible body masses may be applied at the immediate time scale using observed life-history covariance to determine if the physiology of organisms in natural populations is optimised by natural selection. That these models fail as realistic predictors of both contingent and inevitable evolution because they predict the long-term evolution of the simple self-replicator, and because their assumption of constant relative fitnesses is falsified empirically. That deterministic frequency-dependent selection by energetic state and density-dependent competitive interactions predicts the long-term evolution of limited reproduction and non-negligible body masses, and that some of the soft constraints in the historical models can be determined by a deterministic selection that incorporates the historical selection framework on an imme- diate time scale where the relative fitnesses are constant by definition. Inevitable evolution by deterministic selection thus seems to be the most realistic hypothesis for the long-term evolution of large body masses and limited reproductive rates.

In this section I continue to compare the long-term predictions for models of historical and deterministic natural selection focussing on a wider spectrum of lifehistories. This is done to show that the problem with predictions that are long-term evolutionarily unstable is widespread in classical life-history theory, and to show that energetic state and density-dependent competitive interactions provide a unifying selection for the evolution of life-histories in general.

\subsection{Multicellular reproducers}

Associated with the evolution of non-negligible body masses there have been evolutionary transitions from replicators to reproducers (Michod 1983, 1999; Szathmáry and Maynard Smith 1997) and from unicellular to multicellular organisms. Replicators are organisms where the heritable code is given by the structure of the phenotype, so that they have no distinction between a genotype and a phenotype. Reproducers, on the other hand, are organisms where the heritable code is only a fraction of the organism with a clear distinction between a genotype and a phenotype.

Replicator coding is extremely inflexible and can only be expected to be able to code for very simple organisms. The argument that reproducers with so-called unlimited heredity is required for biotic evolution (Szathmáry and Maynard Smith 1997), however, is an overstatement as a set of different self-replicator types, with possibilities of mutational jumps from one type to the other, would allow some evolution to take place. But for the evolution of large organisms with organised phenotypes we need first the evolution of reproducers with unlimited heredity because heritable coding for large and organised phenotypes requires a heritable code that is flexible and independent of the phenotype. Selection pressures for size and increased phenotypic organisation will thus automatically induce selection for a solution to the problem of limited heredity, and the evolution of reproducers is the obvious phenotypic solution.

The evolution of multicellularity requires cooperation at the higher multicellular level to trade-off fitness at the cellular level for increased fitness at the multicellular level (Buss 1987; Michod 1996, 1997, 1999; Michod and Roze 2001). And just like the evolution of reproducers can be seen as a necessity for the evolution of size and phenotypic organisation, so can multicel- 
lularity be seen as a lower level trait that is required for the evolution of large and phenotypically organised organisms. It appears to be impossible for large organisms to be physiologically and functionally efficient without some sort of internal functional differentiation and organisation. Internal differentiation and organisation is thus a natural response to selection for increased size under the constraint that current levels of behaviour and/or external structure must be maintained for the larger organisms to have a selective advantage. The multicellular organism is an obvious solution to this problem because the interactive behaviours of the whole organism trade off fitness at the cellular level for increased fitness at the multicellular level.

\subsection{Pre-reproductive period and interactive behaviour}

The evolution of a prolonged pre-reproductive period, i.e. the period from birth to age at maturity (first reproductive event), is somewhat unexpected because early maturing organisms have faster population growth from reproduction early in life (Cole 1954; Hamilton 1966) and from a higher probability of surviving to maturity (Bell 1980).

Beginning with Gadgil and Bossert (1970) delayed maturity has been suggested to evolve because the benefits of early maturation trade off against a fitness cost of early maturation if fecundity increases with age of maturation (e.g. Wiley 1974; Stearns and Crandall 1981), if fecundity increases with size and size increases with age of maturation (e.g. Schaffer 1974; Bell 1980; Charlesworth 1980; Roff 1981, 1984, 1986; Ziòlko and Kozlowski 1983; Stearns and Koella 1986; Kozlowski and Wiegert 1987; Kozlowski and Uchmanski 1987; Charnov 1993), or if delayed maturity implies increased offspring investment with a sufficiently large increase in the intrinsic survival of the offspring (e.g. Wiley 1974; Stearns and Crandall 1981; reviewed by Kozlowski 1992; Roff 1992; Stearns 1992).

While the historical selection models mentioned above may use observed covariance between the age of maturation and other life-history characters to examine if natural organisms have a physiology that is optimised by natural selection, the models do not identify the ultimate causes for the evolution of delayed maturation. Nearly all the models assume that fecundity increases proportionally with size, as observed within many species and assumed also by historical models on the evolution of body mass. The increase in fecundity with size, however, generally does not hold between closely related species (e.g. Roff 1982), and this shows that the relationship cannot be a hard constraint. When instead the relationship between reproduction and body mass is exchanged with the inverse relation that is expected by hard energetic constraints (eqn 9) the evolutionary expectation is the absence of delayed maturation.

The classical models on delayed maturity are also questioned empirically by interspecific variation, where the pre-reproductive period generally scales to the positive 1/4-power of body mass (Peters 1983). Thus, with the intrinsic Malthusian parameter scaling to the negative 1/4-power of body mass (Fenchel 1974), the prereproductive period is not surprisingly inversely related to the intrinsic Malthusian parameter. Hence, there is no evidence for a general positive correlation across species between the pre-reproductive period and the intrinsic Malthusian parameter as we should expect if the period evolves from historical selection with constant relative fitnesses. It is therefore most likely that the pre-reproductive period evolves by some form of frequency-dependent selection.

Frequency-dependent selection by density-dependent competitive interactions suggests that a prolonged prereproductive period evolves as an interactive trait that allows the offspring to learn intelligent interactive behaviour. If interactive selection on behaviour favours the ability to out-smart other individuals during competition, the individuals with the most developed behavioural skills will have the potential to out-compete the individuals with less developed behaviour. And as time is required to learn and adjust flexible behaviour there may be selection for the pre-reproductive period to become an interactive trait that is required for the development of intelligent interactive behaviour. Witting (2003a) showed that this can indeed be the case with a prolonged pre-reproductive period being expected in high-energy organisms only, and the ratio of the pre-reproductive period to the potential lifespan being an increasing function of the importance of learned behaviour in interactive competition. As confirmed empirically by interspecific comparisons the intrinsic Malthusian parameter is predicted to be negatively correlated with the pre-reproductive period.

\subsection{Metabolic rate}

Although 'the essential phenotypic feature of the first living things was metabolism' given as 'linked series of chemical reactions, driven by an extrinsic source of energy' (Maynard Smith and Szathmáry 1995, p. 18), there is almost no treatment of metabolism in relation to life-history evolution. Not a single model 
on metabolic rate is presented in the life-history reviews of Charlesworth $(1980,1994)$, Roff $(1992,2002)$, Stearns (1992), Bulmer (1994) and Stearns and Hoekstra (2000). The evolution of metabolism has instead been considered mainly at the molecular level and typically in relation to the origin of life (e.g. Miller 1953; Haldane 1954; Oparin and Clark 1959; Ponnamperuma and Chela-Flores 1993; Chela-Flores et al. 1995; Baltscheffsky 1996).

The metabolic rate of mobile organisms is correlated with the intrinsic Malthusian parameter that defines the traditional frequency-independent fitness measure for historical natural selection. Fenchel (1974) showed that the intrinsic Malthusian parameter scales to the negative 1/4-power of body mass, and Southwood (1981) suggested that this relation arose from a similar scaling of the relative metabolic rate and its known negative correlation with longevity. These relationships indicate a possible selection for a continued increase in metabolic rate if the frequency-independent fitness definition is to be taken literally. Frequency-independent fitness, however, seems unrealistic in this case because Cope's rule (Cope 1871, 1887) and between-species comparisons suggest that larger organisms, with lower metabolic rates and smaller intrinsic Malthusian parameters, generally evolve from smaller organisms, with higher metabolic rates and larger intrinsic Malthusian parameters. This would be impossible by frequencyindependent selection where Fisher's Fundamental Theorem of Natural Selection determines that the intrinsic Malthusian parameter can only increase during evolution by natural selection. For the intrinsic Malthusian parameter to decline by natural selection, selection needs to be frequency-dependent.

A frequency- and density-dependent life-history model on the evolution of metabolic rate is provided by deterministic selection by energetic state and densitydependent competitive interactions (Witting 2003a). Just as interactive competition may select for the body mass to become an interactive trait, it may also select for the metabolic rate per unit body mass to become an interactive trait, where the individuals with the highest metabolic rate can be selected to allocate more energy into competitive interactions than individuals with lower metabolic rates. In low-energy organisms where interactive competition is almost absent, the energetic trade-off between metabolism and the traits of survival and reproduction selects for a minimum metabolism, while the level of interactive competition in high-energy organisms is sufficiently large to select for an increased metabolic rate that is used by individuals to monopolise resources during competitive encounters with other

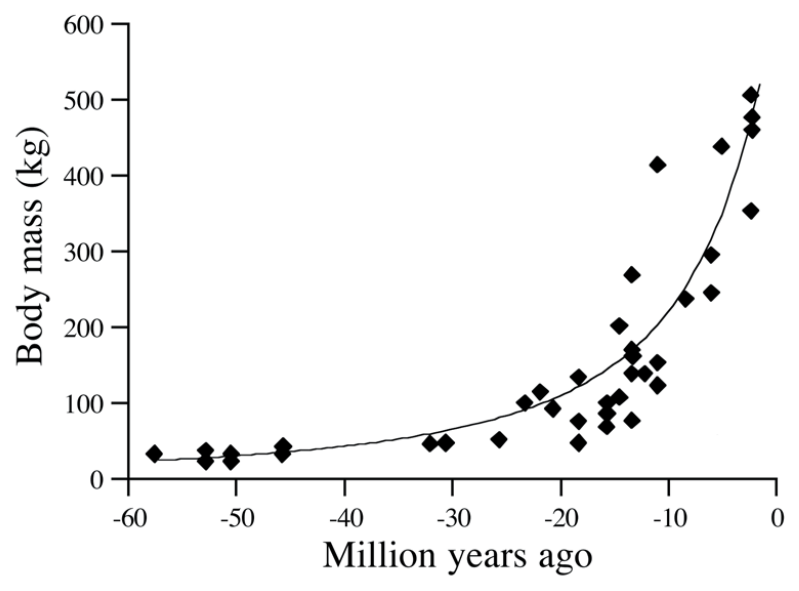

Figure 2: The evolutionary body mass trajectory for fossil horses during the last 57 million years. Points are data from MacFadden (1986), and the curve a fitted trajectory for the evolutionary steady-state with an exponentially increasing body mass. From Witting (1997).

individuals in the population (Witting 2003a). And at the evolutionary steady-state with a continuously increasing body mass the metabolic rate may either be stable at an evolutionary equilibrium or increasing almost exponentially (Witting 2003a). The curvature of the evolutionary increase in the body mass of fossil horses (Fig. 2), for example, has been found to indicate that the metabolic rate of the horse is ten times higher today than it was 57 million years ago (Witting 1997; data from MacFadden (1986), corrected for a body mass increase from $\approx 25 \mathrm{~kg}$ to $\approx 500 \mathrm{~kg}$ over the same period of time).

\subsection{Body mass allometries}

Body mass allometries $\left(q \propto w^{\beta}\right)$ are empirically confirmed relationships that describe phenotypic and ecological parameters $(q)$ as power functions of body mass $(w)$, with the exponents $(\beta)$ having characteristic values for different traits (see reviews by Peters 1983; Calder 1984; Glazier 2005).

Since Rubner (1883) proposed that an allometric exponent of $2 / 3$ for the metabolic rate reflects a surface rule of thermal homeostasis body mass allometries have been seen as reflections of intrinsic constraints. The intrinsic bounds on metabolic rate are often assumed to be so fundamental that they determine not only a single unique exponent for the allometry of metabolism, but also unique exponents for other phenotypic and ecologically related allometries (e.g. Kooijman 1993; Brown et al. 2004; van der Meer 2006). 
The surface rule, however, ran into problems when Kleiber (1932), Brody and Proctor (1932) and Benedict (1938) found that the across-species exponent for metabolic rate was more likely to be $3 / 4$ than $2 / 3$, and nor could the rule explain that the exponent for endotherms and ectotherms is similar (but see Bejan 2001). But the intrinsic hypothesis remained the preferred explanation with new proposals suggesting that metabolic scaling may reflect a surface rule in four spatial dimensions (Blum 1977), resource uptake and use at cell or body surfaces (Davison 1955; Patterson 1992; Makarieva et al. 2003), tissue demands for resources (McMahon 1973; Darveau et al. 2002), resource demand with cellular and demographic constraints (Kozlowski and Weiner 1997; Kozlowski et al. 2003a,b), resource demand and exchange (Sibly and Calow 1986; Kooijman 2000; Banavar et al. 2002a,b), intrinsic constraints on resource transportation systems (West et al. 1997, 1999a,b; Banavar et al. 1999; Dodds et al. 2001; Dreyer and Puzio 2001; Rau 2002; Santillán 2003), thermodynamic constraints at the molecular level (Fujiwara 2003), quantum mechanical constraints on proton and electron flow in metabolic pathways (Demetrius 2003, 2006), or scaling in the four dimensions of space and time (Ginzburg and Damuth 2008).

Among the intrinsic models some are based solely on physical, chemical or geometrical constraints (Rubner 1883; McMahon 1973; Patterson 1992; Rau 2002; Fujiwara 2003) while others also include evolutionary optimisation of the physiology, for example, by minimisation of energy costs (West et al. 1997; Santillán 2003) or fluid volume (Banavar et al. 1999) in distribution networks, minimisation of heat-flow resistance (Bejan 2001), or maximisation of resource-exchange surfaces (West et al. 1999b). The model of Kozlowski et al. $(2003 a, b)$ considers allometries in a broader framework of body mass evolution caused by the positive relationship between body mass and reproductive rate.

Being based on historical selection for body mass it is unlikely that the model of Kozlowski et al. (2003a,b) will explain the long-term evolution of body mass allometries (see Section III.3), and nor might most of the intrinsic hypotheses. Most of the intrinsic models on the evolution of allometric exponents are historical models where the so-called constraints that are used to determine the allometric scaling are phenotypic traits that evolve by natural selection. The model of West et al. (1997), for example, assumes that the terminal units of the resource transportation system are independent of body mass, whereas the radius and length of the capillaries do in fact evolve with body mass so that they are larger in larger animals (Dawson 2003). Most al- lometric hypotheses based on intrinsic geometrical factors furthermore predict that the allometric exponents should be identical within and across natural species (Kozlowski and Weiner 1997; Witting 1998; West et al. 2001; Bokma 2004). This, however, is not always the case (see e.g. Peters 1983 and Calder 1984); the exponent for lifetime reproduction is often independent of body mass across species and proportional to body mass within a species. It is also essential to note that analysis of intraspecific allometries shows that it is unlikely that there is one single universal body mass scaling for metabolic rate, let it be $2 / 3,3 / 4$ or any other number (Bokma 2004).

Frankino et al. (2005) have shown that the allometric scaling of morphological traits is indeed determined by natural selection instead of being given exclusively by developmental, physical, chemical or geometrical constraints. This indicates that allometric exponents evolve to reflect the allometric constraints on the natural selection process itself. Ecological selection may thus be essential because this would allow the exponents to differ among species groups that are exposed to different ecological conditions. Given the four dimensions of ecological space and physiological time, it has been shown that the exponents of eight interspecific body mass allometries can evolve from the allometric constraints on a foraging process that is optimised by natural selection (Witting 1995). The foraging efficiency of an individual is limited not only by the overall exploitation of the population, but more essentially by the local resource exploitation of that individual and by the density-dependent competitive interactions among individuals. Optimal foraging will thus require that both excessive interactive competition and excessive local resource exploitation be minimised so that the individual can obtain the largest amount of resource.

The allometric exponents that follow from this natural selection optimisation of the foraging process include the $3 / 4$ exponent for metabolic rate, the negative $1 / 4$ exponent for the intrinsic Malthusian parameter, and the negative $3 / 4$ exponent for population abundance (Witting 1995, 1997). Hence, the observed negative relationship between body mass and the classical definition of fitness is explained by a selection that is based on density-dependent competitive interactions. Other intriguing predictions include the exponent for lifetime reproduction that generally is independent of body mass across species and proportional to body mass within a species. And that the numerical values of the $1 / 4$ and $3 / 4$ exponents do not hold universally; they are instead suggested to depend on the details of the foraging process. The $1 / 4$ and $3 / 4$ exponents should generally 


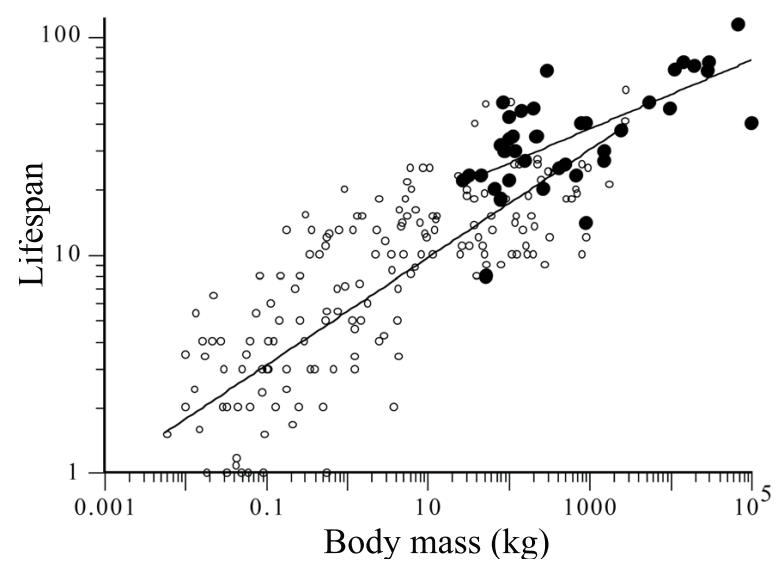

Figure 3: The body mass allometry for lifespan among 195 species of terrestrial mammals where the mean exponent, \pm S.E.M., is $0.25 \pm 0.04$ (open circles), and among 40 species of marine mammals where the exponent is $0.16 \pm 0.02$ (solid circles). From Witting (1997), data from Nowak (1991).

hold for organisms that forage in two spatial dimensions, while the corresponding exponents should be $1 / 6$ and $5 / 6$ for organisms that forage in three spatial dimensions. This prediction is confirmed for terrestrial mammals that forage in two spatial dimensions and marine mammals that forage in three spatial dimensions (Fig. 3).

\subsection{Senescence and soma}

Senescence is a deterioration of tissue with age that causes the age-specific survival probabilities and fecundities to decline with age. While all non-negligible-sized organisms appear to be senescing, they could, at least in principle, have evolved a degree of tissue self-repair that would allow for potential immortality.

Since Weismann (1889) senescence has been seen as a contingent character that evolves from the intrinsic constraints of a soma that is not passed on in either sexual or asexual reproduction. Somatic organisms have a decline in the force of selection with age (Medawar 1946, 1952; Hamilton 1966), and this implies that senescence can evolve by the accumulation of deleterious mutations because this would affect mainly the older age classes where the force of selection is weak (Medawar 1952; Edney and Gill 1968; Charlesworth 1990; Partridge and Barton 1993). Alternatively senescence can evolve by antagonistic pleiotropy where age-structured tradeoffs favour mutations that increase survival and/or fecundity in the younger age classes at the cost of survival and/or fecundity in the older age classes (Williams
1957; Kirkwood 1977, 1990; Charlesworth 1980; Templeton 1980; Rose 1985; Kirkwood and Rose 1991). While senescence by deleterious mutation accumulation seems almost inevitable given reasonable mutation rates and a supply of age-specific mutations (Charlesworth 1990; Partridge and Barton 1993), the evolution of senescence by antagonistic pleiotropy requires that the fitness benefits of enhanced early survival/reproduction are larger than the costs of reduced late survival/reproduction.

The difficulty with senescence, however, it seems, is not to explain its evolution, but rather to explain why it apparently did not evolve in simple self-replicatorlike organisms. The traditional explanation is that agestructured demography applies only to organisms with a soma so that the Malthusian parameter, and the decline in force of selection with age, cannot be calculated from the age structure of an asomatic organism (Rose 1991). As asomatic organisms tend to reproduce by binary fission it is impossible to distinguish the "mother" from the "offspring" and we should therefore be unable to determine the age structure of the demographic rates for an individual with no soma. This conclusion, however, is true only if the demographic rates of the "mother" and the "offspring" differ. But in asomatic organisms with binary fission the two rates are statistically the same and, thus, the age-structured demography of an asomatic evolutionary lineage is easily defined by its reproduction and survival rate, and potential for infinite individual lifespan. The decline in the force of selection with age will therefore apply to both asomatic and somatic organisms, and this indicates that soma and the declining force of selection with age are not the essential components that determine that some organisms have senescence while others do not.

Noting instead that senescence cannot evolve without a clear separation between a senescing somatic tissue and a non-senescing reproductive tissue, Witting (1997) proposed that the soma is the trait through which senescence is expressed. Selection for senescence simply implies that there is selection for the expression of senescence in a soma and for the absence of senescence in a germ line. If this division is not the case the senescing organisms will have zero fitness because the senescing tissue of the mother will be passed on to her offspring, and both the mother and her offspring will die from senescence at approximately the same time. Such lineages cannot persist and nor can they evolve by natural selection.

The critical point left is then a dichotomy that will explain the absence of senescence in simple selfreplicator-like organisms and the presence of senescence 
in organisms at higher energetic states. If senescence does not follow inevitably from the decline in the force of selection with age, one possible mechanism would be that the cost of self-repair increases disproportionately with size and/or phenotypic organisation (e.g. the cost of self-repair in DNA relative to the number of genes) so that the decline in the force of selection with age could favour increased early reproduction and survival in phenotypically organised organisms, while in simple organisms the cost of self-repair would be below a threshold that would allow full-blown self-repair to evolve. Senescence and soma may also evolve by density-dependent competitive interactions that operate independently of the decline in the force of selection with age. Here, the higher level of interactive competition in high-energy organisms can select for the allocation of energy into interactive quality at the cost of self-repair, while in low-energy organisms with no interactive competition selection can favour self-repair at the cost of interactive quality (Witting 1997).

Alternatively it might be that the decline in the force of selection with age is always selecting for the evolution of senescence and soma, but that senescence and soma simply cannot evolve in organisms below a certain level of organisation. Organisms at a sufficiently low energetic state might have a physiology and genetic coding that are so simple that they cannot express, or code for, age-specific effects, and nor may they easily be selected to do so. The apparent absence of senescence in simple self-replicator-like organisms may thus not reflect natural selection, but only a constraint of evolutionary simplicity. A distinction between potential immortality by natural selection or simplicity is that potential immortality should be maintained actively by some degree of self-repair if it evolves by natural selection, while it should be a passive trait that reflects the absence of age-related processes if it is given by simplicity.

\subsection{Sexual reproduction}

Sexual reproduction is reproduction where gametes fuse to form a single zygote that develops into an offspring that contains the joint heritable variation of all gametes. Usually we think of sexual reproduction as the haploid-diploid cycle, where haploid gametes from a diploid male and a diploid female fuse to form a diploid offspring. But higher level sexual reproduction is also in principle possible where $m-1$ haploid gametes from $m-1 m$-ploid males fuse with a haploid gamete from an $m$-ploid female to form an $m$-ploid offspring $(2 \leq m<\infty)$.

The evolution of sexual reproduction has remained one of the most enduring problems in evolutionary biology. The problem is that the usual form of sexual reproduction between a male and a female is four times more costly than asexual reproduction (Witting 1997, $2002 b$ ). The cost of sex is fourfold because there is a twofold cost of meiosis (Williams 1975, 1988), where the probability that an allele of a gene is transferred from a parent to an offspring is reduced from one in asexuals to one-half in sexuals, and a twofold cost of the male (Maynard Smith 1968, 1978), where non-replication in males implies that females on average need to produce two offspring instead of one in order to produce a reproducing unit. More generally, sexual reproduction between $m$ individuals is $2 m$-fold as costly, because the female needs to produce $m$ individuals on average to form a reproducing unit, while at the same time the probability that an allele is transferred to a specific offspring is $1 / m$.

Sexual reproduction is currently seen as a contingent character that evolves from a historical selection imposed by genetic recombination. So far at least twenty different hypotheses have been proposed (for reviews see Bulmer 1994; Kondrashov 1994; Ebert and Hamilton 1996; Hurst and Peck 1996; Otto and Lenormand 2002; Agrawal 2006; Otto and Gerstein 2006), and according to these sexual recombination may protect against the accumulation of deleterious mutations, for example, by Muller's ratchet where recombination breaks up genetic associations between loci allowing for selective elimination of deleterious mutations (Fisher 1930; Muller 1932, 1964; Crow and Kimura 1965; Kondrashov 1982, 1993; Manning and Thompson 1984; Wagner and Gabriel 1990; Charlesworth et al. 1993; Lynch et al. 1993; Peck 1994; Lynch et al. 1995; Peck et al. 1997; Peck et al. 1999), by segregation that breaks up genetic associations within a locus (Otto 2003; Dolgin and Otto 2003), by evolutionary traction where deleterious mutations in asexual organisms hitchhike to fixation owing to selection for rare beneficial mutations (Manning and Thompson 1984; Rice 1987; Hadany and Feldman 2005), or by Hill-Robertson interference that reduces the effective population size making Muller's ratchet more effective (Hill and Robertson 1966; Felsenstein and Yokoyama 1976; Palsson 2002; Keightley and Otto 2006). Recombination has also been seen to favour sexual reproduction because it brings together favourable alleles from different chromosomes, which may increase the rate of adaptation (Weismann 1889; Kodric-Brown and Brown 1987; Kondrashov 1993; Barton 1995; Davis 1995; Jaffé 1996, 1999; Feldman et al. 1997). This conclusion, however, seems to hold only if fitnesses are strictly additive between loci. If not, as it 
is often the case even with two loci, the asexual lineage will generally adapt faster than the sexual lineage (Kondrashov and Kondrashov 2001). Sexual recombination has also been seen as an advantage in biotic interactions, where the Red Queen hypothesis suggests that the diploid genome can hide genes that currently are bad but can be exposed later by recombination when they have become beneficial, for example as protection against new interactions with mutant parasites (Hamilton 1980; Bell 1982; Bell and Maynard Smith 1987; Hamilton et al. 1990; Ebert and Hamilton 1996). More recent studies, however, have shown that interspecific interactions more typically act against the evolution of sex (Otto and Nuismer 2004; Agrawal and Otto 2006; Gandon and Otto 2007).

A widespread limitation to the genetic hypotheses is that they do not generate direct selection that can outbalance the cost of sex and select for the evolution of sexual reproduction. Most of the genetic models will provide only a long-term advantage to the sexual population over the asexual population, and this advantage cannot generally explain the evolutionary transition from asexual to sexual reproduction, nor the evolutionary maintenance of sexual reproduction should asexual variants arise in sexual populations (for exceptions see Kondrashov 1982, 1993; Peck et al. 1999). Another limitation to most of the genetic models is that they do not consider why asexual reproduction is more common in negligible-sized organisms than in larger organisms, nor why the sexual unit is a single male per female with the average offspring receiving half of the genes from the father and the other half from the mother. Instead the models often predict that only a small degree of sex at a low frequency is fine (Green and Noakes 1995; Hurst and Peck 1996; but see Peck and Waxman 2000; Keightley and Otto 2006), and this suggests that the relatively rare gene exchange that occurs among haploid and asexually reproducing prokaryotes is sufficient to account for most of the genetic hypotheses.

While a truly convincing recombination advantage that can outbalance the fourfold cost of sexual reproduction has not yet been identified, the real problem with the contingent hypothesis is that sexual reproduction does not follow from genetic recombination. Although recombination occurs in almost all organisms with sexual reproduction, recombination is not an intrinsic part of the haploid-diploid cycle that defines sexual reproduction. The two traits are therefore not constrained to co-occur with one-another, and there is no fixed link between them as illustrated by sexual reproduction with no recombination in microsporidia (Raikov 1982; Canning 1988) and recombination in the asexual worker reproduction of the Cape honeybee, Apis mellifera capensis (Verma and Ruttner 1983). Selection for genetic recombination in itself is therefore expected to select, not for sexual reproduction, but only for recombination in an asexually reproducing organism.

Instead of using the logically problematic argument of explaining one trait (sexual reproduction) from the fitness advantage to another independent trait (genetic recombination), models on the evolution of genetic recombination would do better if they focussed on a fitness advantage that would outbalance the cost of recombination, i.e. the cost of breaking apart co-adapted gene combinations. In the same way, using the direct fitness trade-off of a trait, models on the evolution of sexual reproduction should outbalance the cost of meiosis by a fitness advantage to the parents sharing of the genome in the offspring, as well as outbalancing the cost of non-replication in the male by a fitness advantage to non-replication.

Like the other fitness costs considered herein the fourfold cost of sexual reproduction is frequencyindependent. This suggests that there is a frequencydependent advantage that can outbalance the fourfold cost, and that this advantage is generated by frequencydependent interactions at the level of the interacting unit that defines the level of selection. For sexually reproducing organisms the interacting unit is likely to be the unit of individuals that may potentially engage in sexual reproduction with one another. At this level density-dependent competitive interactions provide direct selection for the presence of sexual reproduction in high-energy mobile organisms with non-negligible body masses, and direct selection for the absence of sexual reproduction in low-energy organisms with negligible body masses (Witting 1997, 2002b).

Selection for increased interactive quality can select for sexual reproduction because interacting units with several co-operating individuals can dominate units with only a single individual, while at the same time the interactive quality of an interacting unit with several individuals can be enhanced if it contains only a single replicating female while the remaining individuals are non-replicating interacting individuals that allocate energy and time into interactive competition at the cost of replication. A female in such a system can enhance the interactive quality of her interacting unit through the introduction of sexual reproduction because this allows her to attract the non-replicating individuals with the largest interactive quality by allowing them to become males that allocate their genetic code into her offspring through sexual reproduction. With sexual reproduc- 
tion and female competition for interacting males, other things being equal, it is the female that allows the male to allocate the largest fraction of his genome to her offspring that can attract the males with the highest interactive quality.

But where is the evolutionary balance of interactive competition when reproduction can occur on the continuum from asexual reproduction to the highest level of sexual reproduction with infinitely many males per female? Theoretical details are given by Witting (2002b), and again it is the evolutionarily determined level of interactive competition that strikes the balance. In lowenergy organisms with an absence of interactive competition there is selection for an interacting unit that contains a single asexual self-replicator. More intriguingly, the predicted level of interactive competition at the evolutionary equilibrium for body mass in high-energy organisms is exactly so high that there is selection for an interacting unit that contains a single male and a single female with a sexual reproduction between them where offspring receive half of the genes from the father and the other half from the mother. And at the evolutionary steady-state with an exponentially increasing body mass, the evolutionary equilibrium to the sexual unit remains the same while the equilibrium to the interacting unit contains an additional interacting individual that is predicted to be an offspring of the reproducing pair instead of an additional male. With sexual reproduction evolving independently of genetic recombination, recombination may then evolve secondarily in sexually reproducing diploids if it has a fitness advantage that can outbalance the cost of breaking apart co-adapted gene combinations.

With the selection model by density-dependent competitive interactions being the only model with persistent direct natural selection by first principles for pairwisely sexually reproducing organisms, it is suggested that the ecological constraints of density-dependent interactive competition may determine the intrinsic composition of the organism all the way down to the genetic architecture of sexual reproduction.

\subsection{Eusocial colonies}

The typical eusocial colony is a large reproducing unit with a single female, a single male, and thousands of non-reproducing and sexually produced offspring workers. The traditional explanation for the eusocial colony is contingent upon the intrinsic constraints of genetics (Hamilton 1963, 1964), where models of inclusive fitness aim to explain why offspring choose to raise siblings instead of raising their own offspring. Hamilton (1964) noted that Hymenoptera can be predisposed to eusociality because of the high relatedness among sisters in species with a haplodiploid genome, and Lacy (1980, 1984) extended the argument to include termites that have asymmetrical inheritance from a sex-linked diploid genome. Trivers and Hare (1976) showed that sex allocation theory is central for this mechanism to work; haplodiploids are predisposed to eusociality only if the sex ratio of the sexual caste of the ancestor is female biased. This may happen through split sex ratios (Grafen 1986), where broods produced at different times (Seger 1983) or conditions (Frank and Crespi 1989) have different sex ratios.

While inclusive fitness is a necessity for selection for offspring that choose to raise siblings, enhanced inclusive fitness by asymmetrical inheritance is unlikely to be the factor that induces selection for eusocial colonies. Another factor seems to be needed for the simple reason that many species with symmetrical inheritance are eusocial and that many species with asymmetrical inheritance are not eusocial. Alexander (1974), Michener and Brothers (1974), and Stubblefield and Charnov (1986) argue that the essential factor is parents that force their offspring to co-operate; Evans (1977) and Andersson (1984) that it is nest building; and Jarvis et al. (1994) that mammalian eusociality may evolve by mutual benefit during ecological windows of opportunity. Forcing by parents is generally not an evolutionarily stable strategy because offspring may run away from home and start breeding on their own. And while nests may protect offspring and hereby enhance the inclusive fitness of raising siblings, the ultimate selecting factor in such cases is not the nest itself but the factor that the nest protects against, i.e. the factor that makes it selectively beneficial to have nests and raise siblings.

For a clear understanding of the selection forces that are needed for the evolution of eusocial colonies it is important to consider the interacting unit that defines the level of selection. For eusocial reproduction the eusocial colony is the most likely interacting unit that interacts with other colonies so that the rate of reproduction among colonies becomes differential. This not only implies that the fitness cost of non-replication in the individual worker needs to be traded-off against a corresponding fitness gain at the level of the eusocial colony, but also that the fitness gain at the colony level needs to be sufficiently large to outbalance the total fitness cost summed over all workers. And with the cost of non-replication being twofold for the male that engages in sexual reproduction, the cost of non-replication over all offspring workers in an eusocial colony is almost infinitely large (Witting 2002b). The evolution 
of eusociality thus requires an almost infinitely large frequency-dependent advantage at the colony level in order to outbalance the frequency-independent cost of non-replication in all workers. Such an advantage can be provided by selection by density-dependent competitive interactions.

The idea that co-operating groups can attend and defend resources more efficiently than individuals or less organised groups, and that eusociality may evolve by intense interactive competition for limited resources has existed for some time (Lin and Michener 1972; Alexander 1974; Evans 1977; Emlen 1991; Duffy et al. 2000). The hypothesis was formalised by Witting $(1997,2002 b)$, who showed that if the level of interactive competition is sufficiently large, as is expected if the body mass is upward evolutionarily constrained relative to the energetic state of the organism (Section IV.2), then selection by density-dependent competitive interactions will select for large interacting units with the evolutionary optimum to the composition of individuals in the unit being a single female, a single male and a large number offspring workers that are sexually produced by the male and female (Witting 2002b). As described in the previous section, the essential selection forces behind this prediction are that larger and more co-ordinated groups generally can dominate smaller and less co-ordinated groups, that groups with interacting individuals (males and offspring workers) can dominate groups with replicators (females), and that replicators can compete for interactors by offering sexual reproduction.

For co-operative breeding and eusocial colonies there is the additional complication that reproducing units with a single replicating female and several interacting individuals allow for the evolution of higher levels of sexual reproduction with more than a single male per female. Thus, for eusocial colonies we need to explain not only why the interacting unit contains thousands of interacting individuals per reproducing female, but also why the interactors include only a single male while the remaining interactors are offspring workers. To explain this we note that the ability of the female to enhance the interactive quality of the interacting unit by attracting additional males to the colony through higher level sexual reproduction is a function of diminishing return because the interactive quality of the males that are already in the unit is transmitted through sexual reproduction to the offspring workers. In result it follows that it is only for the initial transition from asexual to pairwise sexual reproduction that the interactive quality of the male will outbalance the cost of sexual reproduction. For all potentially remaining transitions to higher levels of sexual reproduction, the transfer of male interactive quality to the sexually produced offspring workers implies that the interactive quality that can be gained by exchanging an offspring worker with an additional sexual male cannot outbalance the extra meiotic cost to sexual reproduction associated with that transition (for detail see Witting 2002b). The evolutionary optimum is then an eusocial colony with a single female, a single male, and a large number of sexually produced offspring workers. This implies that kin selection by offspring workers becomes the evolutionary result of large interacting units that are selected for by ecological interactions rather than being the factor that induces their evolution.

The ecological interactions in eusocial systems may play an even larger role in determining the intrinsic composition of the colonies and their individuals. Historical selection models on sex ratio evolution in eusocial colonies use the worker sex ratio and the degree of sexual reproduction asymmetry to predict the sex ratio of the sexual caste (Trivers and Hare 1976). These models have now been integrated with colony-level selection by the ecological interaction of pair formation between the sexual male and female in termites, and the absence of pair formation in eusocial Hymenoptera (Witting 1997, 2007). In this framework pair formation in termite-like eusocial systems induces symmetrical selection for a diploid genome and a worker caste with male and female offspring, while the absence of a pair bond in Hymenoptera-like eusocial systems induces asymmetrical selection for a haplodiploid genome and a worker caste with female offspring only.

As the proposed model is more parsimonious than traditional models, i.e. explaining more traits from fewer assumptions, as it provides selection by first principles that outbalance the infinite cost of eusocial reproduction, and as it is part of a general selection principle that can explain evolution from the asexual selfreplicator to sexual eusocials, it is suggested that ecological interactions may ultimately determine not only the evolution of eusocial systems and their composition of individuals, but also their degree of sexual reproduction asymmetry including the genomic ploidy level of the individuals.

\subsection{Sessile organisms}

The models of density-dependent competitive interactions developed above describe mobile organisms where individuals can co-operate in their competition for spatially distributed resources. The interacting unit that defines the level of selection may thus evolve beyond the 
individual if individuals co-operate in their interactive competition. This contrasts to sessile organisms that compete for resources that are provided by a flowing medium, which implies that they compete by position and size in the resource flow. In result, sessile organisms cannot generally co-operate in interactive competition and the interacting unit is constrained to levels at and below the individual organism.

This difference in the mode of interactive competition between mobile and sessile organisms seems to be essential for the evolution of the sexual reproduction dichotomy where reproduction tends to occur among hermaphrodites in sessile organisms while it tends to occur between males and females in mobile organisms. In contrast to dioecious organisms with males and females, hermaphrodites can avoid the fourfold cost of sex. They evidently avoid the twofold cost of the male, and they may also avoid the twofold cost of meiosis. As hermaphrodites reproduce through both the female and male function they can, on average, get one copy of a gene through to the next generation for each offspring produced by the hermaphrodite, avoiding the twofold cost of meiosis where, on average, two offspring are needed to copy a gene to the offspring generation. The widespread dichotomy with dioecious and hermaphroditic sexual reproduction in mobile versus sessile organisms may thus be caused by a natural selection that can outbalance the costs of sex in mobile organisms but not in sessile organisms.

Such a distinction exists by density-dependent competitive interactions, where selection can outbalance the costs of sex in mobile organisms because the cooperative act of sexual reproduction between the male and female increases the interactive quality of the interacting unit of the reproducing pair. In sessile organisms, however, the male cannot compete for the female unless he will also compete against her, and with the interacting unit being constrained at the individual level sexual reproduction cannot evolve as a co-operative trait between males and females. The evolutionary optimum of competitive interactions to sexual reproduction in sessile organisms is thus hermaphroditic individuals that avoid the costs to sex in sessile dioecious organisms.

In relation to general differences in the mode of reproduction between sessile and mobile organisms we may also note that there is no male choice at the individual level in hermaphroditic sessile organisms to prevent asexual reproduction and self-fertilisation to evolve in order to enhance the intrinsic growth rate of the hermaphrodite. This may explain why asexual reproduction and self-fertilisation are widespread in sessile sexual organisms, while these traits are rare in mobile sexual organisms.

\section{Major life-history transitions}

Life-history theory should at best be developed not in isolation trait by trait, but as a unified whole that considers all traits and allows for predictions of major lifehistory transitions. Noting the span of evolutionary diversity of life on Earth it is evident that the major transitions have included only some species. Evolutionary models should thus be sufficiently flexible to explain not only the major transitions but also why they have included only some species. In this section I analyse the consistency between the major transitions and the lifehistory models of historical and deterministic natural selection.

\subsection{Models of historical selection}

Maynard Smith and Szathmáry (1995) proposed that an increase in biotic complexity has been achieved by a series of major evolutionary transitions, where entities capable of independent replication before the transition can only replicate as part of a larger whole afterwards. These transitions should not be regarded as the inevitable result of some general law; a higher level transition should instead be seen as an evolutionary possibility given transitions at lower levels. Agreeing that transitions should be explained in terms of immediate selective advantage, they noted that inclusive fitness by genetic similarity between the lower level replicating units is an important necessity at the time of a transition, while later the higher level unit may be maintained by contingent irreversibility and/or by central control.

Genetic similarity will generally not explain a transition by itself. First of all there needs to be a higher level selection that is strong enough to outbalance the fitness cost of the transition at the lower level. Nor can contingent irreversibility and/or central control be expected to prevent back-transitions to lower level units, although these factors may extend the time that it takes for a mutation to occur to a lower level unit that is selectively beneficial. Take, for example, sexual reproduction in mammals. Here, the general lack of parthenogenesis may imply that there are no asexual-like reproducing variants around among mammals at present, but given sufficient time we expect that some kind of asexual reproducer will arise by mutation in some species. The long-term evolutionary maintenance of a fitnesscostly higher level character like sexual reproduction is 


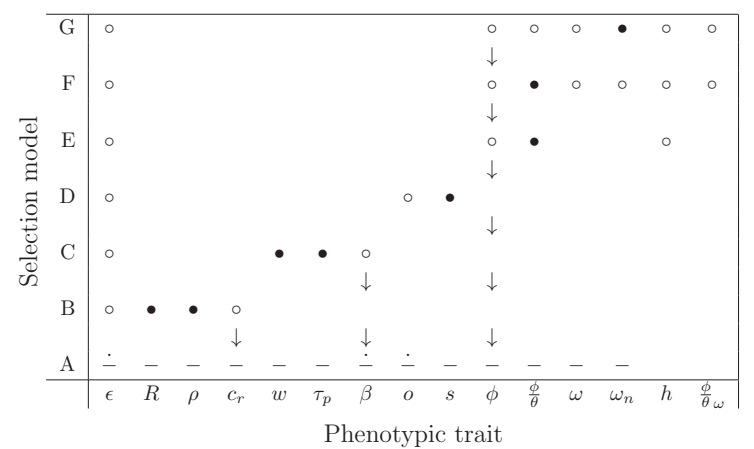

Figure 4: Illustration of the phenotypic structure of historical life-history theory, as it is described by seven independent selection models on the evolution of: A the initial asexual self-replicator; B limited lifetime reproduction and nonnegligible offspring investment; $\mathrm{C}$ non-negligible body mass and a prolonged pre-reproductive period; D senescence; E sex ratio in ordinary species; F sex ratio in eusocial species; $\mathrm{G}$ eusocial reproduction. An open circle (o) is a phenotypic trait that is given as a soft constraint by assumption, a filled circle $(\bullet)$ a trait that is predicted by a model, a dash the absence, or minimum value, of a trait as predicted $(-)$, or assumed $(\dot{-})$, for the initial self-replicator, and no symbol indicates that a trait is not considered for a model. Arrows indicate an expected long-term transition in a soft constraint that is evolutionarily unstable, with the end-prediction of the model being the initial self-replicator. The phenotypic traits of the models are: from the left: $\epsilon$ energetic state; $R$ lifetime reproduction; $\rho$ offspring investment; $c_{r}$ soft trade-off between current reproduction and future survival; $w$ body mass; $\tau_{p}$ pre-reproductive period; $\beta$ allometric exponent of intrapopulation relationship between body mass and reproductive rate; $o$ soma; $s$ senescence; $\phi$ sexual reproduction; $\frac{\phi}{\theta}$ sex ratio; $\omega$ sexually produced offspring workers; $\omega_{n}$ eusocial reproduction; $h$ sexual reproduction asymmetry; $\frac{\phi}{\theta} \omega$ worker sex ratio. Model references: B: Lack (1947); C: Charlesworth (1980) and Roff (1981); D: Williams (1957) and Hamilton (1966); E: Fisher (1930); F: Trivers and Hare (1976); G: Hamilton (1964, 1972).

only guaranteed if there is persistent selection for it.

To examine the existence of persistent selection for higher level units, a summary of the relationship between the historical and deterministic predictions of historical selection models is illustrated for several lifehistories in Fig. 4. It shows the relationship between the soft historical constraints and the predicted traits for some of the most widely accepted models on lifehistory evolution, including models on body mass and reproductive age, reproductive rate and offspring investment, senescence, sex ratio, and eusocial reproduction. Comparing across the different models it is evident that the proposed historical selection on lifehistories is described by models that are independent of one another, with each model explaining a single or a few life-history traits from a set of soft constraints on other traits. Classical life-history theory on the traits of the major evolutionary transitions is not a coherent whole based on a single or a few unifying forces of selection, but instead a diverse collection of hypotheses that are based on different types of selection.

Most of the examined models of historical selection are evolutionarily unstable in their assumptions predicting long-term transitions to negligible sized selfreplicators if the soft constraints are allowed to evolve by the implicit selection of the models. It is a rather general feature of historical selection models that they may use observed relationships to examine if the lifehistory characters of interest are in evolutionary equilibrium, while at the same time they cannot easily explain their long-term evolution. In relation to major transitions the historical models fail on the scale of longterm evolution, where they generally predict the evolution of the starting point for the transitions only, i.e. the evolutionary maintenance of the initial self-replicator.

\subsection{Models of deterministic selection}

A summary of the life-history predictions of the deterministic models of selection by energetic state and density-dependent competitive interactions is given in Fig. 5. Compared with the structure for historical selection (Fig. 4), deterministic selection represents a parsimoniously integrated whole that explains more traits from fewer assumptions. Nearly all the traits in Fig. 5 are explained by selection for interactive quality by density-dependent competitive interactions independently of soft constraints, and the four phenotypic states are evolutionarily stable because they are evolutionarily maintained by a higher level selection that outbalances the lower level cost of the higher level unit. The four phenotypic states are the low-energy self-replicator with a negligible body mass and asexual reproduction, and three states of high-energy organisms with non-negligible body masses, senescence and pairwise sexual reproduction. Dependent upon the resource ecology and body mass constraints the reproducing unit for the latter organism may be the sexual pair, the cooperative unit or the eusocial colony.

Evolutionary transitions between the four phenotypic states are induced by transitions in the evolutionarily determined level of interactive competition in the population. As described in Section IV.2, this level may equilibrate at four states represented by the near absence of interactive competition $\left(\iota^{* i}\right)$ in populations of low-energy organisms, the equilibrium level $\left(\iota^{* *}\right)$ in populations of high-energy organisms with body masses in evolutionary equilibrium, the steady-state level $\left(\iota^{* s}\right)$ in populations of high-energy organisms with exponen- 


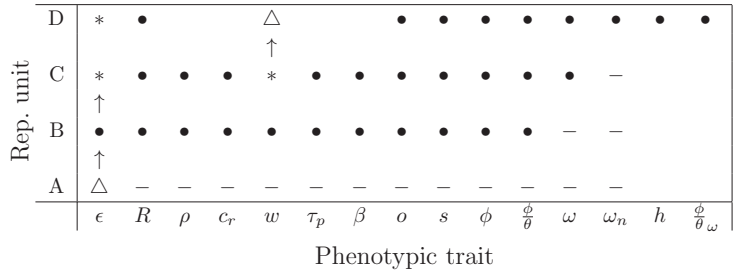

Figure 5: Illustration of the phenotypic structure of deterministic life-history theory by selection by energetic state and density-dependent competitive interactions, as it is described by four reproducing (Rep.) units: A the asexually reproducing self-replicator; $\mathrm{B}$ the sexually reproducing pair; $\mathrm{C}$ the co-operatively reproducing unit; $\mathrm{D}$ the eusocially reproducing colony. A filled circle $(\bullet)$ is a trait that is predicted to be in evolutionary equilibrium, an asterisk $(*)$ a trait that is predicted to be in an evolutionary steady-state of exponential increase, a triangle $(\triangle)$ a trait that is constrained blocking an otherwise predicted increase, and a dash $(-)$ the predicted absence of a trait. An arrow indicates a trait-transition that induces an overall transition between two phenotypic states. Symbols for the phenotypic traits are defined in Fig. 4. Selection models from Witting (1997, 2002b, 2003a, 2007).

tially increasing energetic states and body masses, and the almost infinite level $\left(\iota^{* c}\right)$ in populations of highenergy organisms with body masses that are upward constrained relative to their energetic state.

Starting from the beginning, the first transition from the low-energy self-replicator is given by an evolutionary increase in energetic state, which leads to increased population growth and increased interactive competition. An increase in energetic state will depend on the presence of a sufficiently high density of available resources. Thus, owing to variation in the densities of available resources, and to interspecific interactive competition where species at higher energetic states may prevent species at lower energetic states from shifting to more resource-rich niches, we may expect that selection for an increased energetic state, which allows for the evolution of a body mass in evolutionary equilibrium, can occur only in some organisms.

For the high-energy organisms that have passed the threshold of interaction that allows for the evolution of body masses in evolutionary equilibrium, or have evolved by speciation from ancestors that passed the threshold, a stabilisation at the $\iota^{* *}$ or $\iota^{* s}$ level of interactive competition will depend upon the evolutionary dynamics of resource exploitation. If the resource dynamics is such that the energetic state of the organism will cease to increase at some level, then the population is expected to stabilise at $\iota^{* *}$, which implies the evolution of a stable body mass and pairwise sexual reproduction between a male and female individual. If instead the resource allows for a free evolutionary in- crease in the energetic state of the organisms, then the population is expected to stabilise at the $\iota^{* s}$ level that allows for the evolution of an exponentially increasing body mass and co-operative breeding involving a sexually reproducing pair and one, or a few, of their sexually produced offspring.

The final potential transition to the $\iota^{* c}$ level of interactive competition that allows for the evolution of the fully evolved eusocial colony will depend on a body mass that is evolutionarily constrained to a lower body mass than expected from the energetic state (Witting $2003 a$ ). As this may not generally be expected for organisms with flexible body masses it is of no surprise that fully evolved eusocial colonies have evolved only in relatively few taxa. Constraints that might induce transitions towards the $\iota^{* c}$ level of interactive competition include an ectoskeleton that might set some upper limit to body size, if not on an absolute scale, then on a relative scale where it may limit the possible rate of body mass increase and impose an increased level of interactive competition if the rate of resource exploitation suddenly increases. Such a scenario might be relevant for the evolution of full-blown eusociality in termites and Hymenoptera, which seem to explore plentiful resources that are relatively easy to collect. The constraint that imposes the transition may also be more ecologically determined; a species may, for example, live in narrow canals where selection may operate against large-sized individuals. The latter scenario could be a reason why eusociality has evolved in mole rats, Bathyergidae, that live in subterranean canals (Jarvis 1981), and in sponge-dwelling shrimps, Synalpheus, that live in sponge canals (Duffy 1996; Duffy et al. 2000). Finally, if the upper constraint on body mass has been temporary only, the eusocial unit may continue to persist by natural selection if the existence of eusociality changes the mode of interactive competition so that the interactive quality of the interacting unit is determined more by the number of individual workers in the unit than by the body mass of each individual.

\section{$7 \quad$ Evolutionary arrow of time}

A time-asymmetry of the Second Law of Thermodynamics says that the entropy of an isolated thermodynamic system can only increase with time, and this defines an arrow of time that distinguishes a more disordered future from the past (Eddington 1928). Biological systems, however, are open thermodynamic systems, and here I discuss how selection by energetic state and density-dependent competitive interactions defines an evolutionary arrow of time that distinguishes a more 
structured and organised future from the past for biological systems.

The idea of an evolutionary arrow arose from nonequilibrium thermodynamics where Schrödinger (1944), Bertalanffy (1952) and Prigogine (1978) described biotic systems as dissipative structures that organise themselves through a continued use of the free energy available to the open thermodynamic system. While this observation seems to hold, not only for individual organisms, but also for biological systems over evolutionary time scales, a mechanism that would explain the arrow was missing. The idea was nevertheless extended into a proposed new evolutionary paradigm where thermodynamics were seen as the driving force of a biological evolution towards increased biotic order (e.g. Wicken 1985, 1998; Brooks and Wiley 1986; Schneider and Kay 1994; Swenson 1997). This approach was widely criticised for its lack of biological relevance (e.g. Campbell 1988; Wicken 1988; Rothman 1989; Jordan 1990; Corning and Kline 1998a,b), noting that 'Any introduction of thermodynamic "trends" in evolution that have no demonstrable basis in reproduction and selection means a regression in non-Darwinian, orthogenetic thinking' (Berry 1995, p. 200).

A new formulation of thermodynamics in biological evolution was suggested by Pross (2003, 2005). He noted that the replication reaction is an extreme expression of kinetic control where the thermodynamic requirements play a supporting, rather than directing, role. This proposal has some resemblance with the deterministic interpretation of the historical selection models described earlier herein. This case certainly defines strong directional selection although, when seen on the scale of major evolutionary trends and transitions, it is generally in the direction towards the initial self-replicator.

\subsection{A historic drift}

That biological evolution on Earth has produced structures of increasing organisation over time has been known to the biological sciences for centuries generating a diverse set of hypotheses for long-term evolutionary trends (see McNamara 1990; McShea 1991, 1998). Lamarck (1809) proposed that organisms are driven towards higher levels of perfection, Cope $(1871,1887)$ and Bonner (1988) that there is an increase in the complexity and size of organisms, and Fisher (1930) that the average fitness as represented by the intrinsic Malthusian parameter can only increase over time. Demetrius (1974) argued that demographic entropy will continue to increase for organisms at population dynamic equi- librium (see also Kowald and Demetrius 2005; Ziehe and Demetrius 2005; Bulmer 2006), Vermeij (1987) that organisms become more energy insensitive through evolutionary "arms races", and Maynard Smith and Szathmáry (1995) that hierarchical complexity has increased in a series of major life-history transitions where 'entities that were capable of independent replication before the transition can replicate only as part of a larger whole after it' (p. 4).

We have throughout this paper seen that the longterm evolution of trends towards larger size and increased phenotypic organisation is not generally explained by historical selection. As concluded by Maynard Smith and Szathmáry (1995, p. 4): 'there is no reason why evolution by natural selection should lead to an increase in complexity' and this applies also to the historical interpretation of Cope's rule (Cope 1871, 1887). This rule that describes a body size increase in the phylogenetic lineages of the fossil record is not only one of the best documented trends of long-term evolution, but it is also the trend that most explicitly has been argued to represent contingent evolution (Stanley 1973; Gould 1988).

Cope's rule does not apply to all species, but it often seems to hold as a minimum principle of size increase in at least the maximal size of a taxon, while the minimum size may be stable or even declining (Stanley 1973; Gould 1988; McKinney 1990; Jablonski 1997). In order to explain this Stanley (1973) proposed and Gould (1988) elaborated the view of evolutionary drift away from the evolutionary origin of negligible size. This would explain the tendency for size increase as a lefttruncated and fitness-neutral body mass distribution that undergoes an increase in variance because evolutionary drift can proceed only in the direction of larger size.

Although the historical interpretation of Cope's rule is both logically self-consistent and in general agreement with data it suffers from the absence of a theoretical selection model that explicitly shows that we should expect no directional selection on body mass. But even if firm theoretical arguments for the absence of directional selection on body mass were given, the historical hypothesis goes against empirical evidence that shows strong selection on body mass. If selection is frequency-independent, then fitness is given by the intrinsic Malthusian parameter where the acrossspecies body mass allometries show that smaller organisms should be much fitter than larger organisms because the intrinsic Malthusian parameter declines with the negative $1 / 4$ power of body mass (Fenchel 1974). While a negative body mass scaling of the intrinsic 
Malthusian parameter holds in most cases, the conclusion that smaller organisms are much fitter than larger organisms, evidently is flawed because it predicts that only negligible-sized organisms should exist. Hence large organisms must necessarily persist because of a directional frequency-dependent selection that is strong enough to outbalance the fitness cost of large organisms by frequency-independent selection. Body mass evolution can therefore not be contingent and neutral, but must be controlled for by strong opposing forces of selection with large body masses evolving in the long run only if there is persistent selection for them.

\subsection{A deterministic arrow}

The analysis in this and other papers (Witting 1997, $2002 b, 2003 a$ ) has shown that on the theoretical side there is indeed a reason to regard body mass evolution as being driven by deterministic directional selection. First, we expect that selection for an increase in the energetic state of the organism puts an arrow, or direction, to biotic evolution. And combined with selection by density-dependent competitive interactions we eventually expect an evolutionary steady-state where frequency-dependent selection for a continued increase in body mass is absorbing a large fraction of the evolutionary increase in energetic state, while the intrinsic Malthusian parameter is declining in accordance with the exponents of the body mass allometries.

Dependent upon particular parameterisations we may also expect a continued increase in the metabolic rate, the pre-reproductive period, and in the organisation of the behavioural interactions between individual organisms. Associated with the initial increase in energetic state and body mass we expect a transition from an asomatic, non-senescing, haploid, and asexually reproducing organism to a somatic, senescing, diploid, and sexually reproducing organism with male and female individuals. And in some sexually reproducing organisms there may be an additional transition into the higher level reproducing unit of eusocial colonies.

With selection by interactive competition the phenotypic traits of interactive quality become primary factors that impose selection for further phenotypic innovation. Selection for increased interactive quality, including size, will impose selection for unlimited heredity, and thereby for the evolution of organisms with a genotype and a phenotype (Michod 1999; Witting $2003 a$ ). It will also impose selection for phenotypic organisation, for example, in the form of cell formation, multicellularity, division of labour among cells, and an increased number of cell types (Witting 2003a; Bon- ner 2006). Given sustained selection for competitive quality in interactive behaviour we may eventually expect intelligent behavioural flexibility, expressed, for example, through Machiavellian intelligence (Byrne and Whiten 1988) in social animals. Selection for interactive behaviour may thus select for neural-like networks, increased brain size, increased communication among individuals, and a prolonged pre-reproductive period where individuals learn to behave. Selection by energetic state and density-dependent competitive interactions can therefore be seen as a self-organising force, where populations of self-replicators in open thermodynamic systems organise the energy flow through reproducing units of increasing energetic states and phenotypic organisation, as represented by major evolutionary trends and transitions.

But how general and inevitable can we expect the evolutionary unfolding to be? Provided that the overall environment is favourable for biotic organisms, and that it provides a continued inflow of energy that is available to and attainable by biotic organisms, a longterm evolutionary increase in the energetic state of at least some organisms must be inevitable. And so must some evolutionary increase in body mass provided that the energetic state can evolve to sufficiently high levels. Although appealing as a general principle that includes all organisms with free access to a stable energetic resource, an eternal increase in body mass with increased energetic state cannot, however, be guaranteed. This is because there is likely to be an upper limit to the rate at which an organism can extract energy from its resources and because the increase in body mass depends, at least to some extent, on the overall interactive environment where the interactive quality of an organism will depend not only on body mass, but also on other traits like metabolic rate and interactive behaviour. With regard to the major evolutionary transitions, the theoretical analysis is more complex and, thus, more sensitive to hidden implicit assumptions. The overall result that it is the evolutionary equilibration of body mass that determines the evolutionarily determined level of interactive competition in the population, that populations fall into different categories of interactive competition depending upon energetic state and possible constraints on body mass, and that these categories of increasing interactive competition generate selection for reproducing units of increasing organisation in terms of number of individuals and individual specialisation into reproducers and interactors, however, seems quite robust to hidden assumptions. Superimposed upon this we can expect inevitable evolution of morphological traits like sensory organs in response to unavoidable selection by 
the physical-chemical environment.

All this depends on free access to a relatively stable and plentiful energetic resource. Population dynamics, interspecific interactions, and co-evolutionary responses in the overall biological system, however, may generally imply that the energy that is available to a population will not be constant over time. The assumption of a steady flow of energy to the population will thus be violated for most populations in biological systems, and this is the major reason why we expect, not a monotonic increase in the size of all species, but instead a diverse pattern covering a large range of body masses with some species having a constant mass while the mass of other species is increasing or declining. Nevertheless, if the inflow of energy to the overall biological system is relatively stable over time, we can expect that the species that are interactively superior have access to a relatively stable amount of energy. Especially the dominant species in a competitive guild, which often are the species with larger body masses, may be more likely to experience a free evolutionary increase in energetic state and body mass because their resource access is unlikely to be controlled and limited by interspecific interactions. We may thus expect a size increase in at least the maximal size of a taxon while the minimum size might be stable or even declining.

Empirical studies have found a widespread positive intrapopulation relationship between body mass and reproductive rate (Peters 1983; Reiss 1989), and this agrees with the finding of Kingsolver and Pfennig (2004) that bigger size is associated with higher fitness in most of the natural populations that have been studied. This indicates that many species are situated close to an evolutionary steady-state with an exponentially increasing body mass. Clear-cut cases where the body size has been increasing exponentially in phylogenetic lineages for millions of years include fossil horses (Fig. 2), and the maximal body mass among animals on Earth has been increasing exponentially over the whole fossil record (Fig. 6). A corresponding increase in the maximal relative brain size of carnivores and ungulates during the Tertiary (Jerison 1973) indicates that an increase in the organisation of behavioural interactions may also be an expected trend.

The time-asymmetry of a time arrow is given by irreversibility, with the asymmetry of the evolutionary arrow for increased energetic states in the competitively superior organisms being based on an open thermodynamic system with a constant influx of energy. If the open system begins to experience a steady decline in the inflow of energy, the time-asymmetry of the biotic system is reversed if the decline in the flow of energy

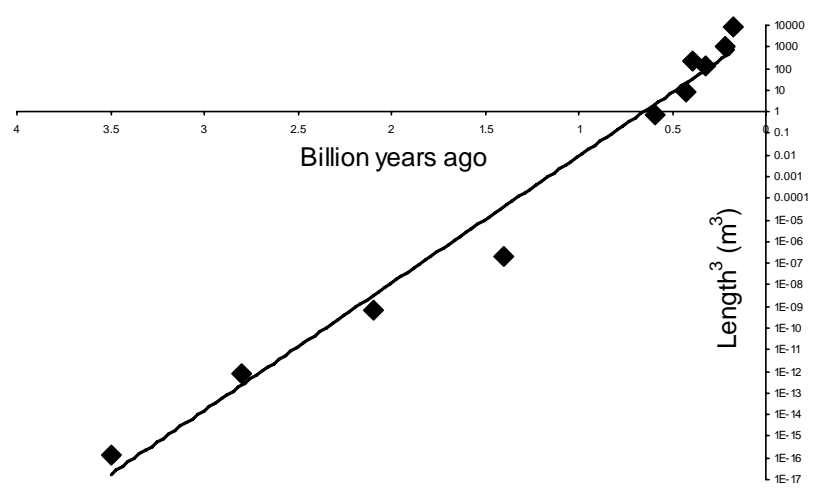

Figure 6: The maximum size (length raised to the third power) of animals in the fossil record over the last 3.5 billion years, and the linear regression that represents an exponentially increasing body mass. Modified from Bonner (1965).

exceeds the evolutionary increase in the ability of the individuals to utilise the resource. A long-term decline in the energetic state of the organisms is then expected, and this implies a decline in the level of interactive competition in populations and a selection for organisms to dwarf (Witting 1997, 2003a), as is often observed in the fossil record during periods of environmental crisis (Guthrie 1984; King and Saunders 1984; Martin and Klein 1984; Barnosky 1989; Donovan 1989; McKinney 1990). A decline in interactive competition caused by a continuously declining resource is expected to affect all interactive traits resulting in a form of backward evolution toward the simple self-replicator at the origin of natural selection. The major evolutionary trends and transitions are thus expected to be reversible, with the overall direction of the evolutionary arrow being defined by the dynamics of the energy flux to the open thermodynamic system.

\section{Conclusions}

(1) To enhance our understanding of life-history evolution we should move from models of contingent evolution by historical natural selection to models of inevitable evolution by deterministic natural selection. Such a change will not deny the role of history and chance in evolution by natural selection, but recognise that the traditional view is incomplete and that contingency plays a more restricted role than previously assumed. While life-history characters and other fitnessrelated traits necessarily must evolve and be evolutionarily maintained by persistent natural selection, neutral characters will most likely depend on history, opportu- 
nity and chance.

(2) It is time to realise that most of the classical lifehistory models that are based on historical natural selection have limited evolutionary applicability. This is because their selection is imposed by soft constraints like genetic covariance, phylogenetic constraints, and phenotypic trade-offs. These constraints evolve by natural selection and when exposed to the implicit selection of the models the assumed constraints tend to be evolutionarily unstable with the predicted long-term equilibrium being the initial self-replicator at the origin of natural selection. The models will thus generally fail as evolutionary predictors; both for contingent evolution, which would require selectively neutral soft constraints, and for inevitable evolution, which would require long-term predictions that differ from the initial self-replicator. Many classical life-history models with constant relative fitnesses are also falsified empirically, because interspecific comparisons show that the relevant fitnesses necessarily must be frequency-dependent. Nevertheless, the classical selection framework can be applied at the immediate time scale where fitnesses are constant per definition. Here it may use the observed life-history co-variance in the population to examine the current evolutionary status of life-history characters.

(3) Inevitable life-history evolution by deterministic natural selection tends to operate by density- and frequency-dependent selection, and it avoids the problem with predictions that are evolutionarily unstable. This is because deterministic selection is imposed independently of soft constraints, being dependent only on fixed constraints and on the ecology of the selfreplication process in limited environments. Models of deterministic selection may thus explain life-history evolution from first principles of self-replication, while they cannot explain phenotypic components that depend on history and initial conditions.

(4) Malthusian Relativity, i.e. the proposed theory of inevitable evolution by deterministic natural selection, is currently the only available theory with deterministic selection for evolutionary transitions from the asexual self-replicator to the sexually reproducing eusocial colony. Given that the physical-chemical conditions are favourable for life, the theory shows that the energetic state of the organism and the density-dependent competitive interactions between individuals in populations select for a life-history transition from the initial self-replicator at the origin of natural selection to large mobile organisms with limited reproductive rates, high metabolic rates, complex behavioural interactions, senescence and pairwise sexual reproduction between a male and a female individual. It furthermore predicts that life-history characters and ecological parameters (including the metabolic rate, intrinsic Malthusian parameter, population density, and home range area) should scale in accordance with the empirically established body mass allometries, and that evolutionary lineages with a body mass that is upward constrained relative to the energetic state can have an additional transition into the fully evolved eusocial colony with thousands of sexually produced offspring workers.

(5) Malthusian Relativity and some classical lifehistory models on the same traits can be seen as dual theories that focus on different aspects of the underlying process of natural selection. This requires that Malthusian Relativity be seen as the theory that operates on the time scale of evolutionary changes, while the framework of classical life-history theory is seen to operate at the immediate time scale where constant relative fitnesses can be used to describe the current selection pressure in the population. In some such cases the deterministic and classical selection models can be combined to explain the evolution of the soft constraints that formed the assumptions of the original version of the classical selection model.

(6) Deterministic selection by density-dependent competitive interactions is tightly inter-linked with population dynamics where it introduces an additional layer of density dependence that is delayed by a single generation. This implies that density-dependent population dynamics evolve into inertia dynamics that can explain the population dynamic cycles that have fascinated ecologists for decades.

(7) The energetic state of the organism implies selection for an evolutionary direction towards organisms of increasing energetic states, and this time-asymmetry defines an evolutionary arrow of time for biotic organisms in open thermodynamic systems with a constant influx of energy. For whole ecosystems, and in line with the fossil record, this implies an evolutionary arrow towards exponential increase in the maximal body mass of a taxon, while the minimum mass might be stable or even declining. Associated with the increase in body mass we can expect an increased phenotypic organisation in accordance with major evolutionary transitions. 
Acknowledgements I am grateful to William Foster and two anonymous reviewers for comments that helped me to improve the article on essential points.

\section{References}

Abrams P. A. Matsuda H. (1994). The evolution of traits that determine ability in competitive contests. Evolutionary Ecology 8:667-686.

Agrawal A. F. (2006). Evolution of sex: Why do organisms shuffle their genotypes? Current Biology 16:696-704.

Agrawal A. F. Otto S. P. (2006). Host-parasite coevolution and selection on sex through the effects of segregation. The American Naturalist 168:617-629.

Alexander R. D. (1974). The evolution of social behavior. Annual Review of Ecology and Systematics 4:325-384.

Andersson M. (1984). The evolution of eusociality. Annual Review of Ecology and Systematics 15:165-189.

Baltscheffsky, H., ed (1996). Origin and Evolution of Biological Energy Conversion. VCH Publishers, New York.

Banavar J. R., Damuth J., Maritan A., Rinaldo A. (2002a). Modelling universality and scaling. Nature 420:626.

Banavar J. R., Damuth J., Maritan A., Rinaldo A. (2002b). Supply-demand balance and metabolic scaling. Proceedings of the National Academy of Sciences of the USA 99:10506-10509.

Banavar J. R., Maritan A., Rinaldo A. (1999). Size and form in efficient transportation networks. Nature 399:130132.

Barnosky A. D. (1989). The late pleistocene event as a paradigm for wide spreadmammal extinction. In: Donovan S. K. (ed). Mass extinctions. Processes and evidence: Columbia University Press, New York, pp 235-254.

Barton N. H. (1995). Linkage and the limits to natural selection. Genetics 140:821-841.

Batzli G. O. (1996). Population cycles revisited. Trends in Ecology and Evolution 11:488-489.

Bejan A. (2001). The tree of convective heat streams: its thermal insulation function and the predicted 3/4power relation between body heat loss and body size. International Journal of Heat Mass Transfer 44:699704.

Bell G. (1980). The cost of reproduction and their consequences. The American Naturalist 110:57-77.

Bell G. (1982). The masterpiece of nature: The evolution of genetics of sexuality. Croom Helm, New York.

Bell G. Maynard Smith J. (1987). Short term selection for recombination among mutually antagonistic species. Nature 328:66-68.

Benedict F. G. (1938). Vital energetics: A study in comparative basal metabolism. Carnegie Institute, Washington DC.
Berry S. (1995). Entropy, irreversibility and evolution. Journal of Theoretical Biology 175:197-202.

Berryman A. A. (1996). What causes population cycles of forest lepidoptera? Trends in Ecology and Evolution 11:28-32.

Bertalanffy L. v. (1952). Problems of life. Watts, London.

Blum J. J. (1977). On the geometry of four-dimensions and the relationship between metabolism and body mass. Journal of Theoretical Biology 64:599-601.

Bokma F. (2004). Evidence against universal metabolic allometry. Functional Ecology 18:184-187.

Bonner J. T. (1965). Size and cycle. Princeton University Press, Princeton.

Bonner J. T. (1988). The evolution of complexity. Princeton University Press, Princeton.

Bonner J. T. (2006). Why size matters. From bacteria to blue whales. Princeton University Press, Princeton.

Boonstra R. Krebs C. J. (1979). Viability of large- and small-sized adults in fluctuating vole populations. Ecology 60:567-573.

Boyce M. S. (1984). Restitution of $r$ - and $K$-selection as a model of density-dependent natural selection. Annual Review of Ecology and Systematics 15:427-448.

Brody S. Proctor R. C. (1932). Relation between basal metabolism and mature body weight in different species of mammals and birds. University of Missouri Agricultural Experiment Station Research Bulletin 116:89-101.

Brooks D. R. McLennan D. A. (1991). Phylogeny, ecology, and behaviour. University of Chicago Press, Chicago.

Brooks D. R. Wiley E. O. (1986). Evolution as entropy. Cambridge University Press, Cambridge.

Brown J. H., Gillooly A. P., Allen V. M., Savage G. B. (2004). Towards a metabolic theory of ecology. Ecology 85:1771-1789.

Brown J. H. Maurer B. A. (1986). Body size, ecological dominance and cope's rule. Nature 324:248-250.

Brown J. S. (1994). Review of Phylogeny, Ecology, and Behaviour by D. R. Brooks and D. A. McLennan. Journal of Mammalogy 75:243-246.

Bulmer M. (1994). Theoretical evolutionary ecology. Sinauer Associates Publishers, Massachusetts.

Bulmer M. (2006). A critique of directionality theory. Proceedings of the Royal Society B 273:635-639.

Buss L. W. (1987). The evolution of individuality. Princeton University Press, Princeton.

Byrne, R. W. \& Whiten, A., eds (1988). Machiavellian Intelligence. Oxford University Press, Oxford.

Calder W. A. I. (1984). Size, function, and life history. Harvard University Press, Cambridge.

Campbell J. H. (1988). Evolution as nonequilibrium thermodynamics: halfway there?. In: Weber B. H., Depew D. J., Smith J. D. (eds). Entropy, information and evolution: The MIT Press, Cambridge, MA, pp 275-284.

Canning E. U. (1988). Nuclear division and chromosome 
cycles in microsporidia. BioSystems 21:333-340.

Case T. J. (1979). Optimal body size and an animal's diet. Acta Biotheoretica 28:54-69.

Charlesworth B. (1971). Selection in density-regulated populations. Ecology 52:469-474.

Charlesworth B. (1980). Evolution in age-structured populations. Cambridge University Press, Cambridge.

Charlesworth B. (1990). Optimizations models, quantitative genetics, and mutation. Evolution 44:520-538.

Charlesworth B. (1994). Evolution in age-structured populations. 2nd edn. Cambridge University Press, Cambridge.

Charlesworth D., Morgan M. T., Charlesworth B. (1993). Mutation accumulation in finite outbreeding and inbreeding populations. Genetical Research 61:39-56.

Charnov E. L. (1993). Life history invariants. Some explorations of symmetry in evolutionary ecology. Oxford University Press, New York.

Charnov E. L. Krebs J. R. (1974). On clutch-size and fitness. Ibis 116:217-219.

Chela-Flores, J., Chadha, M., Negrón-Mendoza, A., \& Oshima, T., eds (1995). Chemical Evolution: SelfOrganization of the Macromolecules of Life. A. Deepak Publishing, Hampton.

Chitty D. (1960). Population processes in the voles and their relevance to general theory. Canadian Journal of Zoology 38:99-113.

Chitty D. (1967). The natural selection of self-regulatory behaviour in animal populations. Proceedings of the Ecological Society of Australia 2:51-78.

Christiansen F. B. (1991). On conditions for evolutionary stability for a continuously varying character. The American Naturalist 138:37-50.

Cole L. C. (1954). The population consequences if life history phenomena. Quartenary Review of Biology 29:103-137.

Conway-Morris S. (2003). Life's solution: Inevitable humans in a lonely universe. Cambridge University Press, Cambridge.

Cooper T. F., Rozen D. E., Lenski R. E. (2003). Parallel changes in gene expression after 20,000 generations of evolution in Escherichia coli. Proceedings of the National Academy of Sciences of the USA 100:1072-1077.

Cope E. D. (1871). The method of creation of organic forms. Proceedings of the American Philosophical Society 12:229-263.

Cope E. D. (1887). The origin of the fittest. D. Appleton and Co, New York.

Corning P. A. Kline J. K. (1998a). Thermodynamics, information, and life revisited, Part 1: to be or entropy. Syst. Res. 15:273-295.

Corning P. A. Kline J. K. (1998b). Thermodynamics, information, and life revisited, Part 2: thermoeconomics and control information. Syst. Res. 15:453-482.

Crow J. Kimura M. (1956). Some genetic problems in nat- ural populations. Proceedings of the Third Berkeley Symposium of Mathematical Statistics and Probability $4: 1-22$.

Crow J. F. Kimura M. (1965). Evolution in sexual and asexual populations. The American Naturalist 99:439450.

Damuth J. (1981). Population density and body size in mammals. Nature 290:699-700.

Damuth J. (1987). Interspecific allometry of population density in mammals and other animals: the independence of body mass and population energy-use. Biological Journal of the Linnean Society 31:193-246.

Darveau C.-A., Suarez K. S., Andrews R. D., Hochachka P. W. (2002). A general model for ontogenetic growth. Nature 417:166-170.

Darwin C. (1859). The origin of species. John Murray, London.

Davis C. H. (1995). The effect of assortative mating and environmental variation on selection for sexual reproduction. Evolutionary Theory 11:51-53.

Davison J. (1955). Body weight, cell surface and metabolic rate in anuran Amphibia. Biological Bulletin 109:407419.

Dawkins R. (2004). The ancestor's tale. A pilgrimage to the dawn of evolution. A Mariner Book, Boston.

Dawkins R. Krebs J. R. (1979). Arms races between and within species. Proceedings of the Royal Society of London: Biological Sciences 205:489-511.

Dawson T. H. (2003). Scaling laws for capillary vessels of mammals at rest and in exercise. Proceedings of the Royal Society of London: Biological Sciences 270:755763.

Day T. Taylor P. D. (1996). Evolutionarily stable versus fitness maximizing life histories under frequencydependent selection. Proceedings of the Royal Society of London: Biological Sciences 263:333-338.

Demetrius L. (1974). Demographic parameters and natural selection. Proceedings of the National Academy of Sciences of the USA 71:4645-4647.

Demetrius L. (2003). Quantum statistics and allometric scaling of organisms. Physica 322:477-490.

Demetrius L. (2006). The origin of allometric scaling laws in biology. Journal of Theoretical Biology 243:455-467.

Dercole F., Ferriére R., Rinaldi S. (2002). Ecological bistability and evolutionary reversals under asymmetrical competition. Evolution 56:1081-1090.

Dieckmann U. (1997). Can adaptive dynamics invade? Trends in Ecology and Evolution 12:128-131.

Dieckmann U. Metz J. A. J. (2006). Surprising evolutionary predictions from enhanced ecological realism. Theoretical Population Biology 69:263-281.

Dodds P. S., Rothman D. H., Weitz J. S. (2001). Reexamination of the "3/4-law" of metabolism. Journal of Theoretical Biology 209:9-27.

Dolgin E. S. Otto S. P. (2003). Segregation and the evolution of sex under overdominant selection. Genetics 
164:1119-1128

Donovan, S. K., ed (1989). Mass extinctions: Processes and evidence. Columbia University Press, New York.

Dreyer O. Puzio R. (2001). Allometric scaling in animals and plants. Journal of Mathematical Biology 43:144156.

Duffy J. E. (1996). Eusociality in a coral-reef shrimp. Nature 381:512-514.

Duffy J. E., Morrison C. L., Rios R. (2000). Multiple origins of eusociality among sponge-dwelling shrimps (Synalpheus). Evolution 54:503-730.

Dugatkin L. A. Reeve H. K. (1998). Game theory and animal behavior. Oxford University Press, Oxford.

Ebert D. Hamilton W. D. (1996). Sex against virulence: the coevolution of parasitic diseases. Trends in Ecology and Evolution 11:79-82.

Eddington A. (1928). The nature of the physical world. MacMillan, London.

Edney E. B. Gill R. W. (1968). Evolution of senescence and specific longevity. Nature 220:281-282.

Emlen S. T. (1991). Evolution of cooperative breeding in birds and mammals. In: Krebs J. R. Davies N. B. (eds). Behavioural ecology: an evolutionary approach. 3d ed.: Blackwell Scientific, Oxford, pp 301-337.

Emlen S. T., Reeve H. K., Sherman P. W., Wrege P. H., Ratnieks F. L. W., Shellman-Reeve J. S. (1991). Adaptive versus nonadaptive explanations of behaviour. The case of alloparental helping. Amazoniana 138:259-270.

Eshel I. (1983). Evolutionary and continuous stability. Journal of Theoretical Biology 103:99-111.

Eshel I. Feldman M. W. (1984). Initial increase of new mutants and some continuity properties of ESS in twolocus systems. Amazoniana 124:631-640.

Eshel I. Feldman M. W. (2001). Optimality and evolutionary stability under short-term and long-term selection. In: Orzack S. H. Sober E. (eds). Adaptationism and Optimality: Cambridge University Press, Cambridge, pp 161-190.

Eshel I., Feldman M. W., Bergman A. (1998). Long-term evolution, short-term evolution, and population genetic theory. Journal of Theoretical Biology 191:391396.

Eshel I. Motro U. (1981). Kin selection and strong evolutionary stability of mutual help. Theoretical Population Biology 19:420-433.

Eshel I., Motro U., Sansone E. (1997). Continuous stability and evolutionary convergence. Journal of Theoretical Biology 185:333-343.

Evans H. E. (1977). Extrinsic versus intrinsic factors in the evolution of insect sociality. BioScience 27:6133-617.

Ewens W. J. (1969). A generalized fundamental theorem of natural selection. Genetics 63:531-537.

Feldman M. W., Otto S. P., Christiansen F. B. (1997). Population genetic perspectives on the evolution of recombination. Annual Review of Genetics 30:261-295.
Felsenstein J. Yokoyama S. (1976). The evolutionary advantage of recombination. II. Individual selection for recombination. Genetics 83:845-859.

Fenchel T. (1974). Intrinsic rate of natural increase: The relationship with body size. Oecologia 14:317-326.

Fisher R. A. (1930). The genetical theory of natural selection. Clarendon, Oxford.

Fisher R. A. (1999). The genetical theory of natural selection. A complete variorum edn. Oxford University Press, Oxford.

Frank S. A. Crespi B. J. (1989). Synergism between sibrearing and sex ratio in Hymenoptera. Behavioral Ecology and Sociobiology 24:155-162.

Frankino W. A., Zwaan B. J., Stern D. L., Brakefield P. M. (2005). Natural selection and developmental constraints in the evolution of allometries. Science 307:718-720.

Fujiwara N. (2003). Origin of the scaling rule for fundamental living organisms based on thermodynamics. BioSystems 70:1-7.

Gadgil M. Bossert P. W. (1970). Life-historical consequences of natural selection. The American Naturalist 104:1-24.

Gandon S. Otto S. P. (2007). The evolution of sex and recombination in response to abiotic or coevolutionary fluctuations in epistasis. Genetics 175:1835-1853.

Geritz S. A. H., Kisdi É., Meszéna G., Metz J. A. J. (1998). Evolutionary singular strategies and the adaptive growth and branching of the evolutionary tree. Evolutionary Ecology 12:35-57.

Geritz S. A. H., Metz J. A. J., Kisdi É., Meszéna G. (1997). Dynamics of adaptation and evolutionary branching. Phys. Rev. Lett. 78:2024-2027.

Gerritsen J. (1984). Size efficiency reconsidered: a general foraging model for free swimming aquatic animals. The American Naturalist 123:450-467.

Ginzburg L. Colyvan M. (2004). Ecological orbits. How planets move and populations grow. Oxford University Press, Oxford.

Ginzburg L. Damuth J. (2008). The space-lifetime hypothesis: viewing organisms in four dimensions, literally. The American Naturalist 171:125-131.

Ginzburg L. R. (1980). Ecological implications of natural selection. In: Barigozzi C. (ed). Vito Volterra symposium on mathematical models in biology. Lecture notes in biomathematics, Vol. 39: Springer-Verlag, Berlin, pp 171-183.

Ginzburg L. R. (1983). Theory of natural selection and population growth. Benjamin Cummings, Menlo Park, CA.

Ginzburg L. R. (1998). Inertial growth. Population dynamics based on maternal effects. In: Mousseau T. A. Fox C. W. (eds). Maternal effects as adaptations: Oxford University Press, New York, pp 42-53.

Ginzburg L. R. Taneyhill D. E. (1994). Population cycles of forest lepidoptera: a maternal effect hypothesis. Jour- 
nal of Animal Ecology 63:79-92.

Glazier D. S. (2005). Beyond the '3/4-power law': variation in the intra- and interspecific scaling of metabolic rate in animals. Biological Reviews 80:611-662.

Godfray H. C. J., Partridge L., Harvey P. H. (1991). Clutch size. Annual Review of Ecology and Systematics 22:409-429.

Gould S. J. (1988). Trends as changes in variance: a new slant on progress and directionality in evolution. Journal of Paleontology 62:319-329.

Gould S. J. (2002). The structure of evolutionary theory. The Belknap Press of Harvard University Press, Cambridge.

Grafen A. (1986). Split sex ratios and the evolutionary origins of eusociality. Journal of Theoretical Biology 122:95-121.

Green R. F. Noakes D. L. G. (1995). Is a little bit of sex as good as a lot? Journal of Theoretical Biology 174:8796.

Guthrie R. D. (1984). Mosaic, allelochemics and nutrients. an ecological theory of late pleistocene megafaunal extinctions. In: Martin P. S. Klein R. G. (eds). Quaternary extinctions. A prehistoric revolution: The University of Arizona Press, Tucson, pp 259-298.

Gyllenberg M. Parvinen K. (2001). Necessary and sufficient conditions for evolutionary suicide. Bulletin of Mathematical Biology 63:981-993.

Hadany L. Feldman M. W. (2005). Evolutionary traction: the cost of adaptation and the evolution of sex. Journal of Evolutionary Biology 18:309-314.

Haigh J. Rose M. R. (1980). Evolutionary game auctions. Journal of Theoretical Biology 85:381-397.

Hairston J. N. G. Hairston S. N. G. (1993). Causeeffect relationships in energy flow, trophic structure, and interspecific interactions. The American Naturalist 142:379-411.

Hairston S. N. G., Smith F. E., Slobodkin L. B. (1960). Community structure, population control, and competition. The American Naturalist 94:421-425.

Haldane J. B. S. (1932). The causes of evolution. Longmans, London.

Haldane J. B. S. (1954). The origins of life. New Biology $16: 12-27$.

Hamilton W. D. (1963). The evolution of altruistic behavior. The American Naturalist 97:354-356.

Hamilton W. D. (1964). The genetical evolution of social behaviour. Journal of Theoretical Biology 7:1-52.

Hamilton W. D. (1966). The moulding of senescence by natural selection. Journal of Theoretical Biology 12:1245 .

Hamilton W. D. (1972). Altruism and related phenomena, mainly in social insects. Annual Review of Ecology and Systematics 3:193-232.

Hamilton W. D. (1980). Sex versus non-sex versus parasite. Oikos 35:282-290.
Hamilton W. D., Axelrod R., Tanese R. (1990). Sexual reproduction as an adaptation to resist parasites. Proceedings of the National Academy of Sciences of the USA 87:3566-3573.

Hammerstein P. (1996). Darwinian adaptation, population genetics and the streetcar theory of evolution. Journal of Mathematical Biology 34:511-532.

Härdling R. (1999). Arms races, conflict costs and evolutionary dynamics. Journal of Theoretical Biology 196:163167.

Harvey, P. A., Brown, A. J. L., Maynard Smith, J., \& Nee, S., eds (1996). New uses from new phylogenies. Oxford University Press, Oxford.

Heino M., Metz J. A. J., Kaitala V. (1998). The enigma of frequency-dependent selection. Trends in Ecology and Evolution 13:367-370.

Hill W. G. Robertson A. (1966). The effects of linkage on limits to artificial selection. Genetical Research 8:269294.

Hofbauer, J. \& Sigmund, K., eds (1998). Evolutionary games and population dynamics. Cambridge University Press, Cambridge.

Holder N. (1983). Developmental constraints and the evolution of vertebrate digit patterns. Journal of Theoretical Biology 104:451-471.

Hurst L. D. Peck J. R. (1996). Recent advances in understanding of the evolution and maintenance of sex. Trends in Ecology and Evolution 11:46-52.

Jablonski D. (1997). Body-size evolution in cretaceous molluscs and the status of cope's rule. Nature 385:250-252.

Jaffé K. (1996). The dynamics of the evolution of sex, why sexes are, in fact, always two? Interciencia 21:259-267.

Jaffé K. (1999). On the adaptive value of some mate selection strategies. Acta Biotheoretica 47:29-40.

Jarvis J. U. M. (1981). Eusociality in a mammal: Cooperative breeding in naked mole-rat colonies. Science 212:571-573.

Jarvis J. U. M., O'Riain M. J., Bennett N. C., Sherman P. W. (1994). Mammalian eusociality: a family affair. Trends in Ecology and Evolution 9:47-51.

Jerison H. J. (1973). Evolution of the brain and intelligence. Academic Press, New York.

Jordan J. C. (1990). Evolution towards increasing entropy according to the Boltzmann model. Evolutionary Theory 9:225-277.

Kauffman S. (1995). At home in the universe. The search for the laws of self-organization and complexity. Oxford University Press, New York.

Kauffman S. A. (1993). The origins of order. Selforganisation and selection in evolution. Oxford University Press, New York.

Keightley P. D. Otto S. P. (2006). Interference among deleterious mutations favour sex and recombination in finite populations. Nature 443:89-92.

Kempthorne O. (1957). An introduction to genetic statistic. Wiley, New York. 
Kimura M. (1958). On the change of population fitness by natural selection. Heredity 12:145-167.

Kimura M. (1965). Attainment of quasi linkage equilibrium when gene frequencies are changing by natural selection. Genetics 52:875-890.

Kimura M. (1978). Change of gene frequency by natural selection under population number regulation. Proceedings of the National Academy of Sciences of the USA 75:1934-1937.

King J. E. Saunders J. J. (1984). Environmental insularity and the extinction of the american mastodont. In: Martin P. S. Klein R. G. (eds). Quaternary extinctions. A prehistoric revolution: The University of Arizona Press, Tucson, pp 315-344.

Kingsolver J. G. Pfennig D. W. (2004). Individual-level selection as a cause of Cope's rule of phylogenetic size increase. Evolution 56:1608-1612.

Kirkwood T. B. L. (1977). Evolution of ageing. Nature 270:301-304.

Kirkwood T. B. L. (1990). The disposable soma theory of aging. In: Harrison D. E. (ed). Genetics effects on aging II: Telford Press, Caldwell, NJ, pp 9-19.

Kirkwood T. B. L. Rose M. R. (1991). Evolution of senescence: late survival sacrificed for reproduction. Philosophical Transactions of the Royal Society of London 332:15-24.

Kisdi É. (1999). Evolutionary branching under asymmetric competition. Journal of Theoretical Biology 197:149 162.

Kleiber M. (1932). Body and size and metabolism. Hilgardia 6:315-353.

Kodric-Brown A. Brown J. H. (1987). Anisogamy, sexual selection, and the evolution and maintenance of sex. Evolutionary Ecology 1:95-105.

Kojima K.-I. Kelleher T. M. (1960). Changes in mean fitness in random mating populations when epistasis and linkage are present. Genetics 46:527-540.

Kondrashov A. S. (1982). Selection against harmful mutations in large sexual and asexual populations. Genetical Research 40:325-332.

Kondrashov A. S. (1993). Classification of hypotheses on the advantage of amphimixis. Journal of Heredity 84:372387.

Kondrashov A. S. (1994). Sex and deleterious mutations. Nature 369:99-100.

Kondrashov F. A. Kondrashov A. S. (2001). Multidimensional epistasis and the disadvantage of sex. Proceedings of the National Academy of Sciences of the USA 98:12089-12092.

Kooijman S. A. L. M. (1993). Dynamic energy budgets in biological systems. Cambridge University Press, Cambridge.

Kooijman S. A. L. M. (2000). Dynamic energy and mass budgets in biological systems. Cambridge University Press, Cambridge.

Kowald A. Demetrius L. (2005). Directionality theory: a computational study of an entropic principle in evolutionary processes. Proceedings of the Royal Society B 272:741-749.

Kozlowski J. (1992). Optimal allocation of resources for age and size at maturity. Trends in Ecology and Evolution 7:15-19.

Kozlowski J., Konarzewski M., Gawelczyk A. T. (2003a). Cell size as a link between noncoding DNA and metabolic rate scaling. Proceedings of the National Academy of Sciences of the USA 100:14080-14085.

Kozlowski J., Konarzewski M., Gawelczyk A. T. (2003b). Intraspecific body size optimization produce interspecific allometries. In: Blackburn T. M. Gaston K. J. (eds). Macroecology: Concepts and consequences: Blackwell, Malden, Massachusetts, pp 299-320.

Kozlowski J. Uchmanski J. (1987). Optimal individual growth and reproduction in perennial species with indeterminate growth. Evolutionary Ecology 1:214-230.

Kozłowski J. Weiner J. (1997). Interspecific allometries are by-products of body size optimization. The American Naturalist 149:352-380.

Kozlowski J. Wiegert R. G. (1987). Optimal age and size at maturity in annuals and perennials with determinate growth. Evolutionary Ecology 1:231-244.

Krebs C. J. (1978). A review of the chitty hypothesis of population regulation. Canadian Journal of Zoology 56:2464-2480.

Krebs C. J. (1996). Population cycles revisited. Journal of Mammalogy 77:8-24.

Krebs C. J. Myers J. (1974). Population cycles in small mammals. Advances in Ecological Research 8:267-399.

Lack D. (1947). The significance of clutch size. Ibis 89:302352.

Lacy R. C. (1980). The evolution of eusociality in termites: A haplodiploid analogy? The American Naturalist 116:449-451.

Lacy R. C. (1984). The evolution of termite eusociality: Reply to Leinaas. The American Naturalist 123:876879.

Lamarck J.-B. (1809). Philosophie zoologique. Paris.

Larson A. Losos J. B. (1996). Phylogenetic systematics of adaptation. In: Roose M. R. Lauder G. V. (eds). Adaptation: Academic Press, San Diego, pp 187-220.

Li C. C. (1955). Population genetics. University of Chicago Press, Chicago.

Liberman U. (1988). External stability and ESS: criteria for initial increase of a mutant allele. Journal of Mathematical Biology 26:477-485.

Lidicker W. Z. Ostfeld R. S. (1991). Extra-large body size in California voles: Causes and fitness consequences. Oikos 61:108-121.

Lin N. Michener C. D. (1972). Evolution of eusociality in insects. Quartenary Review of Biology 47:131-159.

Lotka A. J. (1925). Elements of physical biology. Williams and Wilkins, Baltimore. 
Lundberg S. Persson L. (1993). Optimal body size and resource density. Journal of Theoretical Biology 164:163180.

Lynch M., Bürger R., Butcher D., Gabriel W. (1993). The mutational meltdown in asexual populations. Journal of Heredity 84:339-344.

Lynch M., Conery J., Bürger R. (1995). Mutational meltdown in sexual populations. Evolution 49:1067-1080.

MacColl A. D. C., Piertney S. B., Moss R., Lambin X. (2000). Spatial arrangement of kin affects recruitment success in young male red grouse. Oikos 90:261-270.

MacFadden B. J. (1986). Fossil horses from "eohippus" (hyracotherium) to equus: scaling, cope's law, and the evolution of body size. Paleobiology 12:355-369.

Makarieva A., Gorshkov V. G., Li B.-L. (2003). A note on metabolic rate dependence on body size in plants and animals. Journal of Theoretical Biology 221:301-307.

Malthus T. R. (1798). An essay on the principle of population. Johnson, London.

Manning J. T. Thompson D. J. (1984). Muller's ratchet accumulation of favorable mutations. Acta Biotheoretica 33:219-225.

Martin, P. S. \& Klein, R. G., eds (1984). Quaternary extinctions. A prehistoric revolution. The University of Arizona Press, Tucson.

Martins E. P. (1996). Phylogenies and the comparative method in animal behaviour. Oxford University Press, Oxford.

Matessi C. Di Pasquale C. (1996). Long-term evolution of multilocus traits. Journal of Theoretical Biology 185:333-343.

Matessi C. Eshel I. (1992). Sex ratio in the social hymenoptera: a population-genetics study of long-term evolution. The American Naturalist 139:276-312.

May R. M. (1976). Simple mathematical models with very complicated dynamics. Nature 261:459-467.

May R. M. Oster G. F. (1976). Bifurcation and dynamic complexity in simple ecological models. Amazoniana 110:573-599.

Maynard Smith J. (1968). Evolution in sexual and asexual populations. The American Naturalist 102:469-473.

Maynard Smith J. (1978). The evolution of sex. Cambridge University Press, Cambridge.

Maynard Smith J. (1982). Evolution and the theory of games. Cambridge University Press, Cambridge.

Maynard Smith J. Brown R. L. W. (1986). Competition and body size. Theoretical Population Biology 30:166-179.

Maynard Smith J. Price G. R. (1973). The logic of animal conflict. Nature 246:15-18.

Maynard Smith J. Szathmáry E. (1995). The major transitions in evolution. W.H. Freeman Spektrum, Oxford.

Mayr E. (1988). Toward a new philosophy of biology. Observations of an evolutionist. Harvard University Press, Cambridge.

McKinney M. L. (1990). Trends in body-size evolution. In:
McNamara K. J. (ed). Evolutionary Trends: The University of Arizona Press, Tucson, pp 75-118.

McKitrick M. C. (1993). Phylogenetic constraint in evolutionary theory: Has it any explanatory power? Annual Review of Ecology and Systematics 24:307-330.

McLaren I. A. (1966). Adaptive significance of large size and long life of the chaetognarth sagitta elegans in the arctic. Ecology 47:852-855.

McMahon T. A. (1973). Size and shape in biology. Science 179:1201-1204

McNamara, K. J., ed (1990). Evolutionary trends. The University of Arizona Press, Tucson.

McShea D. W. (1991). Complexity and evolution: What everybody knows. Biology and Philosophy 6:303-324.

McShea D. W. (1998). Possible largest-scale trends in organismal evolution: Eight "live hypothesis". Annual Review of Ecology and Systematics 29:293-318.

Medawar P. B. (1946). An unsolved problem of biology. Modern Quarterly 1:30-56.

Medawar P. B. (1952). An unsolved problem of biology. Lewis, London.

Metz J. A. J., Geritz S. A. H., Meszéna G., Jacobs F. J. A., vanHeerwaarden J. S. (1996a). Adaptive dynamics, a geometrical study of the consequences of nearly faithful reproduction. In: vanStrien S. J. Verduyn Lunel S. M. (eds). Stochastic and spatial structures of dynamical systems: North Holland, Amsterdam, The Netherlands, pp 183-231.

Metz J. A. J., Mylius S. D., Diekmann O. (1996b). When does evolution optimize? On the relation between types of density dependence and evolutionary stable life history parameters. IIASA WP:96-04.

Metz J. A. J., Nisbet R. M., Geritz S. A. H. (1992). How should we define fitness' for general ecological scenarios? Trends in Ecology and Evolution 7:198-202.

Michener C. D. Brothers D. J. (1974). Were workers of eusocial Hymenoptera initially altruistic or oppressed? Proceedings of the National Academy of Sciences of the USA 71:671-674.

Michod R. E. (1983). Population biology of the first replicators: on the origin of the genotype, phenotype and organism. American Zoologist 23:5-14.

Michod R. E. (1996). Cooperation and conflict in the evolution of individuality. II. Conflict mediation. Proceedings of the Royal Society of London: Biological Sciences 263:813-822.

Michod R. E. (1997). Cooperation and conflict in the evolution of individuality. I. Multilevel selection of the organism. The American Naturalist 149:607-645.

Michod R. E. (1999). Darwinian dynamics. Evolutionary transitions in fitness and individuality. Princeton University Press, Princeton.

Michod R. E. Roze D. (2001). Cooperation and conflict in the evolution of multicellularity. Heredity $86: 1-7$.

Miller S. L. (1953). A production of amino acids under possible primitive Earth conditions. Science 117:528- 
529.

Muller H. J. (1932). Some genetic aspects of sex. Nature 66:118-138.

Muller H. J. (1964). The relation of recombination to mutational advance. Mutation Research 1:2-9.

Mylius S. D. Diekmann O. (1995). On evolutionarily stable life histories, optimization and the need to be specific about density dependence. Oikos 74:218-224.

Nicholson A. J. (1960). The role of population dynamics in natural selection. In: Tax S. (ed). Evolution after Darwin: Vol. 1. The evolution of life: University of Chicago Press, Chicago, pp 477-520.

Nisbet R. M. Gurney W. S. C. (1982). Modelling fluctuating populations. John Wiley, Chichester, U. K.

Norrdahl K. (1995). Population cycles in northern small mammals. Biological Reviews 70:621-637.

Nowak R. M. (1991). Walker's mammals of the world volume I-II. 5th edn. The Johns Hopkins University Press, Baltimore.

Oparin, A. I. \& Clark, F., eds (1959). The origin of life on Earth. Pergamon Press, New York.

Oster G. F. (1976). Internal variables in population dynamics. In: Levin S. (ed). Lectures on Mathematics in the Life Sciences, vol. 8: American Mathematical Society, Providence, RI, pp 37-68.

Otto S. P. (2003). The advantage of segregation and the evolution of sex. Genetics 164:1099-1118.

Otto S. P. Gerstein A. C. (2006). Why have sex? The population genetics of sex and recombination. Biochemical Society Transactions 34:519-522.

Otto S. P. Lenormand T. (2002). Resolving the paradox of sex and recombination. Nature Reviews, Genetics 3:252-261.

Otto S. P. Nuismer S. L. (2004). Species interactions and the evolution of sex. Science 304:1018-1020.

Palsson S. (2002). Selection on a modifier of recombination rate due to linked deleterious mutations. Journal of Heredity 93:22-26.

Parker G. A. (1979). Sexual selection and sexual conflict. In: Blum M. S. Blum N. A. (eds). Sexual selection and reproductive competition in insects: Academic Press, New York, pp 123-166.

Parker G. A. (1983). Arms races in evolution-an ess to the opponent-independent cost game. Journal of Theoretical Biology 101:619-648.

Partridge L. Barton N. H. (1993). Optimality, mutation and the evolution of ageing. Nature 362:305-311.

Patterson M. R. (1992). A mass transfer explanation of metabolic scaling relations in some aquatic invertebrates and algae. Science 255:1421-1423.

Peck J. R. (1994). A ruby in the rubbish: beneficial mutations and the evolution of sex. Genetics 137:597-606.

Peck J. R., Barreau G., Heath S. C. (1997). Imperfect genes, Fisherian mutation and the evolution of sex. Genetics 145:1171-1199.
Peck J. R. Waxman D. (2000). What's wrong with a little sex? Journal of Evolutionary Biology 13:63-69.

Peck J. R., Yearsley J., Barreau G. (1999). The maintenance of sexual reproduction in a structured population. Proceedings of the Royal Society of London: Biological Sciences 266:1857-1864.

Pelosi L., Kühn L., Guetta D., Garin J., Geiselmann J., Lenski R. E., Schneider D. (2006). Parallel changes in global protein profiles during long-term experimental evolution in Escherichia coli. Genetics 173:1851-1869.

Peters R. H. (1983). The ecological implication of body size. Cambridge University Press, Cambridge.

Ponnamperuma, C. \& Chela-Flores, J., eds (1993). Chemical Evolution: Origin of Life. A. Deepak Publishing, Hampton.

Price G. R. (1972). Fisher's "fundamental theorem" made clear. Annals of Human Genetics 36:129-140.

Prigogine I. (1978). Time, structure, and fluctuations. Science 201:777-785.

Pross A. (2003). The driving force for Life's emergence: Kinetic and thermodynamic considerations. Journal of Theoretical Biology 220:393-406.

Pross A. (2005). On the emergence of biological complexity: life as a kinetic state of matter. Origins of Life and Evolution of Biospheres 35:151-166.

Raikov I. B. (1982). The protozoan nucleus. SpringerVerlag, Heidelberg and New York.

Rau A. R. P. (2002). Biological scaling and physics. Journal of Bioscience 27:475-478.

Reeve H. K. Sherman P. W. (1993). Adaptation and the goals of evolutionary research. Quartenary Review of Biology 68:1-32.

Reeve H. K. Sherman P. W. (2001). Optimality and phylogeny: A critique of current thought. In: Orzack S. H. Sober E. (eds). Adaptation and optimality: Cambridge University Press, Cambridge, pp 64-113.

Reiss M. J. (1989). The allometry of growth and reproduction. Cambridge University Press, Cambridge.

Rice W. R. (1987). Genetic hitchhiking and the evolution of reduced genetic activity of the $\mathrm{Y}$ sex chromosome. Genetics 116:161-167.

Ricklefs R. E. (1990). Ecology. 3th edn. W.H. Freeman \& Company, New York.

Ridely M. Thompson D. J. (1979). Size and mating in asellus aquaticus (crustacea: Isopoda). Z. Tierpsychol. 51:380-397.

Roff D. A. (1981). On being the right size. The American Naturalist 118:405-422.

Roff D. A. (1982). Reproductive strategies in flatfish: a first synthesis. Canadian Journal of Fisheries and Aquatic Sciences 38:968-977.

Roff D. A. (1984). The evolution of life history parameters in teleosts. Canadian Journal of Fisheries and Aquatic Sciences 41:989-1000.

Roff D. A. (1986). Predicting body size with life history 
models. BioScience 836:316-323.

Roff D. A. (1992). The evolution of life histories. Theory and analysis. University of Chicago Press, New York.

Roff D. A. (2002). Life history evolution. Sinauer Associates, Inc., Massachusetts.

Rose M. R. (1985). Life history evolution with antagonistic pleiotropy and overlapping generations. Theoretical Population Biology 28:342-358.

Rose M. R. (1991). Evolutionary biology of aging. Oxford University Press, New York.

Rothman T. (1989). Science à la mode. Princeton University Press, Princeton.

Roughgarden J. (1971). Density-dependent natural selection. Ecology 5:453-468.

Rubner M. (1883). Über den einfluss der korper grosse auf stoff-und kraft-wechsel. Z. Biol. 19:535-562.

Saccheri I. Hanski I. (2006). Natural selection and population dynamics. Trends in Ecology and Evolution 21:341-347.

Salthe S. N. (1989). Untitled. In: Hecht M. K. (ed). Evolutionary biology at the crossroads: Queens College Press, New York, pp 174-176.

Santillán M. (2003). Allometric scaling law in a simple oxygen exchanging network: possible implications on the biological allometric scaling laws. Journal of Theoretical Biology 223:249-257.

Schaffer W. M. (1974). Selection for optimal life histories: the effect of age structure. Ecology 55:291-303.

Schaffer W. M. (1983). The application of optimal control theory to the general life history problem. The American Naturalist 121:418-431.

Scheider E. D. Kay J. J. (1994). Life as a manifestation of the second law of thermodynamics. Math. Comput. Model. 19:25-48.

Schoener T. W. (1969). Models of optimal size for solitary predators. The American Naturalist 103:277-313.

Schrödinger E. (1944). What is life? Cambridge University Press, Cambridge.

Seger J. (1983). Partial bivoltinism may cause alternating sex-ratio biases that favour eusociality. Nature 301:5962.

Segre A. v., Murray A. W., Leu J. Y. (2006). High-resolution mutation mapping reveals parallel experimental evolution in yeast. PLOS Biology 4:1372-1385.

Sibly R. M. Calow P. (1986). Physiological ecology of animals. Blackwell, Oxford.

Simpson G. G. (1953). The major features of evolution. Columbia University Press, New York.

Sinclair A. R. E. (1989). Population regulation in animals. In: Cherrett J. M. (ed). Ecological concepts. The contribution of ecology to an understanding of the natural world: Blackwell Scientific Publications, Oxford, pp 197-241.

Sinervo B., Svensson E., Comendant T. (2000). Density cycles and an offspring quantity and quality game driven by natural selection. Nature 406:985-988.

Southwood T. R. E. (1981). Bionomic strategies and population parameters. In: May R. M. (ed). Theoretical ecology: Sinauer, Sunderland, MA, pp 30-52.

Stanley S. M. (1973). An explanation for cope's rule. Evolution $27: 1-26$.

Stearns S. C. (1992). The evolution of life histories. Oxford University Press, Oxford.

Stearns S. C. Crandall R. E. (1981). Quantitative predictions of delayed maturity. Evolution 35:455-463.

Stearns S. C. Hoekstra R. F. (2000). Evolution: an introduction. Oxford University Press, Oxford.

Stearns S. C. Koella J. (1986). The evolution of phenotypic plasticity in life-history traits: Predictions for norms of reaction for age- and size-at-maturity. Evolution 40:893-913.

Stenseth N. C. (1982). Causes and consequences of dispersal in small mammals. In: Swingland I. Greenwood P. (eds). The ecology of animal movement: Oxford University Press, Oxford, pp 62-101.

Stenseth, N. C. \& Ims, R., eds (1993). The biology of lemmings. Academic Press, San Diego.

Stubblefield J. W. Charnov E. L. (1986). Some conceptual issues in the origin of eusociality. Heredity 57:181-187.

Swenson R. (1997). Thermodynamics, evolution, and behavior. In: Greenberg G. Haraway M. (eds). The encyclopedia of comparative psychology: Garland, New York, pp 217-227.

Szathmáry E. Maynard Smith J. (1997). From replicators to reproducers: the first major transitions leading to life. Journal of Theoretical Biology 187:555-571.

Taylor P. D. (1989). Evolutionary stability in one-parameter models under weak selection. Theoretical Population Biology 36:125-143.

Templeton A. R. (1980). The evolution of life histories under pleiotropic constraints. Theoretical Population Biology 18:279-289.

Trivers R. L. Hare H. (1976). Haplodiploidy and the evolution of social insects. Science 191:249-263.

van derMeer J. (2006). Metabolic theories in ecology. Trends in Ecology and Evolution 21:136-140.

Van Valen L. (1973). A new evolutionary law. Evolutionary Theory 1:1-30.

Vega-Redondo F. (1996). Evolution, Games, and Economic Behaviour. Oxford University Press, Oxford.

Verma S. Ruttner F. (1983). Cytological analysis of the thelytokous parthenogenesis in the Cape honeybee (Apis mellifera capensis Escholts). Apidologie 14:41-57.

Vermeij G. J. (1987). Evolution and escalation. Princeton University Press, Princeton.

Vermeij G. J. (1996). Adaptations of clades: Resistance and response. In: Roose M. R. Lauder G. V. (eds). Adaptation: Academic Press, San Diego, pp 363-380.

Vincent T. L. Brown J. S. (1988). The evolution of ess theory. Annual Review of Ecology and Systematics 
19:423-443.

Volterra V. (1926). Variazioni e fluttuazioni del numero d'individui in specie animali conviventi. Mem. Acad. Lincei. 2:31-113.

Wagner G. P. Gabriel W. (1990). Quantitative variation in finite parthenogenetic populations: what stops Muller's Ratchet in the absence of recombination? Evolution 44:715-731.

Weismann A. (1889). Essays upon heredity and kindred biological problems. Clarendon Press, Oxford.

West G. B., Brown J. H., Enquist B. J. (1997). A general model for the origin of allometric scaling laws in biology. Science 276:122-126.

West G. B., Brown J. H., Enquist B. J. (1999a). A general model for the structure and allometry of plant vascular systems. Nature 400:664-667.

West G. B., Brown J. H., Enquist B. J. (1999b). The fourth dimension of life: Fractal geometry and allometric scaling of organisms. Science 284:1677-1679.

West G. B., Brown J. H., Enquist B. J. (2001). A general model for ontogenetic growth. Nature 413:628-631.

Wicken J. S. (1985). Thermodynamics and the conceptual structure of evolutionary theory. Journal of Theoretical Biology 117:363-383.

Wicken J. S. (1988). Thermodynamics, evolution and emergence: Ingredients for a new synthesis. In: Weber B. H., Depew D. J., Smith J. D. (eds). Entropy, information and evolution: The MIT Press, Cambridge, MA, pp 159-168.

Wicken J. S. (1998). Evolution and thermodynamics. The new paradigm. Syst. Res. 15:365-372.

Wiegert R. G. Owen D. F. (1971). Trophic structure, available resources and population density in terrestrial vs. aquatic ecosystems. Journal of Theoretical Biology 30:69-81.

Wiley R. H. (1974). Effects of delayed reproduction on survival, fecundity, and the rate of population increase. The American Naturalist 108:705-709.

Williams G. C. (1957). Pleiotropy, natural selection and the evolution of senescence. Evolution 11:398-411.

Williams G. C. (1966). Adaptation and natural selection. A critique of some current evolutionary thought. Princeton University Press, Princeton.

Williams G. C. (1975). Sex and evolution. Princeton University Press, Princeton.

Williams G. C. (1988). Retrospect on sex and kindred topics. In: Michod R. E. Levin B. R. (eds). The Evolution of Sex: Sinauer, Sunderland, pp 287-298.

Witting L. (1995). The body mass allometries as evolutionarily determined by the foraging of mobile organisms. Journal of Theoretical Biology 177:129-137.

Witting L. (1997). A general theory of evolution. By means of selection by density dependent competitive interactions. Peregrine Publisher, Århus, 330 pp, URL http://mrLife.org.

Witting L. (1998). Body mass allometries caused by phys- iological or ecological constraints? Trends in Ecology and Evolution 13:25.

Witting L. (2000a). Interference competition set limits to the fundamental theorem of natural selection. Acta Biotheoretica 48:107-120.

Witting L. (2000b). Population cycles caused by selection by density dependent competitive interactions. Bulletin of Mathematical Biology 62:1109-1136.

Witting L. (2002a). Evolutionary dynamics of exploited populations selected by density dependent competitive interactions. Ecological Modelling 157:51-68.

Witting L. (2002b). From asexual to eusocial reproduction by multilevel selection by density dependent competitive interactions. Theoretical Population Biology 61:171-195.

Witting L. (2002c). Two contrasting interpretations of Fisher's fundamental theorem of natural selection. Comments on Theoretical Biology 7:1-10.

Witting L. (2003a). Major life-history transitions by deterministic directional natural selection. Journal of Theoretical Biology 225:389-406.

Witting L. (2003b). Reconstructing the population dynamics of eastern Pacific gray whales over the past 150 to 400 years. The Journal of Cetacean Research and Management 5:45-54.

Witting L. (2007). Behavioural interactions selecting for symmetry and asymmetry in sexual reproductive systems of eusocial species. Bulletin of Mathematical Biology 69:1167-1198.

Wooton R. J. (1979). Energy cost of egg production and environmental determinants of fecundity in teleost fishes. Symposia of the Zoological Society of London 44:133 159.

Wright S. (1930). Review of fisher (1930). Journal of Heredity $21: 349-356$.

Wright S. (1931). Evolution in mendelian populations. Genetics 16:97-159.

Wright S. (1955). Classification of the factors of evolution. Cold Spring Harbour Symposium on Quantitative Biology 20:16-24.

Wynne-Edwards V. C. (1962). Animal dispersion in relation to social behavior. Oliver \& Boyd, Edinburgh.

Wynne-Edwards V. C. (1986). Evolution through group selection. Blackwell Scientific Publications, Oxford.

Wynne-Edwards V. C. (1993). A rationale for group selection. Journal of Theoretical Biology 162:1-22.

Ziehe M. Demetrius L. (2005). Directionality theory: an empirical study of an entropic principle in life-history evolution. Proceedings of the Royal Society B 272:11851194.

Ziòlko M. Kozlowski J. (1983). Evolution of body size: an optimization model. Mathematical Bioscience 54:127143. 


\section{A Appendix}

\begin{tabular}{|c|c|}
\hline & Life-history selection symbols \\
\hline$c_{r}$ & $\begin{array}{l}\text { Soft trade-off between current reproduction } \\
\text { and future survival }\end{array}$ \\
\hline$c_{w}$ & Soft trade-off between survival and body mass \\
\hline$f$ & $\begin{array}{l}\text { Density regulation function, approximated } \\
\text { as } N^{-\gamma}\end{array}$ \\
\hline$h$ & Sexual reproduction asymmetry \\
\hline$m$ & Ploidy level of genome \\
\hline$N$ & Population density \\
\hline$p$ & Survival; probability to survive to reproduce \\
\hline$q$ & Unspecified trait \\
\hline$r$ & Malthusian parameter (exponential growth rate) \\
\hline$R$ & Lifetime reproduction \\
\hline$s$ & Senescence \\
\hline$w$ & Adult body mass \\
\hline$\epsilon$ & $\begin{array}{l}\text { Energetic state; lifetime net energy for } \\
\text { reproduction and growth }\end{array}$ \\
\hline$\epsilon_{R}$ & $\begin{array}{l}\text { Net energy for lifetime reproduction, } \\
\epsilon_{R}=\epsilon-(1-\rho) w\end{array}$ \\
\hline$\gamma$ & Total density regulation, $\gamma=\gamma_{\alpha}+\mu \gamma_{\iota}$ \\
\hline$\gamma_{\alpha}$ & Density regulation by exploitation \\
\hline$\gamma_{\iota}$ & $\begin{array}{l}\text { Density dependence in number of competitive } \\
\text { interactions }\end{array}$ \\
\hline$\iota$ & $\begin{array}{l}\text { Level of interactive competition among } \\
\text { interacting units (often individuals) }\end{array}$ \\
\hline$\gamma_{q}$ & $\begin{array}{l}\text { Inertia; delayed density dependence caused by } \\
\text { natural selection, } \gamma_{q}=\gamma_{\iota} \psi \sigma^{2}\end{array}$ \\
\hline$\lambda$ & $\begin{array}{l}\text { Discrete Malthusian parameter (per } \\
\text { generation growth rate) }\end{array}$ \\
\hline$\mu$ & $\begin{array}{l}\text { Individual cost, or payoff, from competitive } \\
\text { interactions }\end{array}$ \\
\hline$\omega$ & Sexually produced offspring workers \\
\hline$\omega_{n}$ & Eusocial reproduction \\
\hline$o$ & Soma \\
\hline$\phi$ & Sexual reproduction \\
\hline$\frac{\phi}{\theta}$ & Sex ratio \\
\hline$\frac{\phi}{\theta}$ & Sex ratio of worker caste in eusocial colonies \\
\hline$\psi^{\theta \omega}$ & $\begin{array}{l}\text { Slope between } \mu \text { and } w \text { across variants in a } \\
\text { population }\end{array}$ \\
\hline$\psi_{\rho}$ & $\begin{array}{l}\text { Slope between } \mu \text { and } \rho \text { across variants in a } \\
\text { population }\end{array}$ \\
\hline$\rho$ & $\begin{array}{l}\text { Parental investment in offspring body mass; } \\
\text { as a fraction of } w\end{array}$ \\
\hline$\sigma^{2}$ & Additive genetic variance of interactive quality \\
\hline$\tau_{p}$ & $\begin{array}{l}\text { Pre-reproductive period } \\
\text { Superscripts }\end{array}$ \\
\hline$\beta$ & Body mass exponent in allometry, $q \propto w^{\beta}$ \\
\hline$\hat{\imath}$ & $\begin{array}{l}\text { Intrinsic trait component (genetically } \\
\text { determined) }\end{array}$ \\
\hline - & $\begin{array}{l}\text { Extrinsic trait component (ecologically } \\
\text { determined) }\end{array}$ \\
\hline$*$ & Population dynamic equilibrium \\
\hline$* *$ & $\begin{array}{l}\text { Evolutionary equilibrium (continuously stable } \\
\text { strategy, CSS) }\end{array}$ \\
\hline$* c$ & Upward constrained evolutionary equilibrium \\
\hline$* i$ & Downward constrained evolutionary equilibrium \\
\hline$* s$ & Evolutionary steady-state \\
\hline & Subscripts \\
\hline$i$ & $i$ th variant in a population \\
\hline$t$ & Time in generations \\
\hline
\end{tabular}

Selection structure symbols (Section 2)

\begin{tabular}{|c|c|}
\hline$E$ & Environment \\
\hline$F$ & Selection function \\
\hline $\mathbf{P}$ & Phenotype (set of phenotypic traits) \\
\hline \multirow[t]{2}{*}{$q$} & Unspecified phenotypic trait \\
\hline & Superscripts \\
\hline $\mathbf{c}$ & $\begin{array}{l}\text { Complete parameter space of all possible } \\
\text { phenotypes }\end{array}$ \\
\hline$i$ & Specific parameterisation (variant) \\
\hline$* \mathbf{i}$ & $\begin{array}{l}\text { Complete initial parameter space at natural } \\
\text { selection origin }\end{array}$ \\
\hline \multirow[t]{2}{*}{$* *$} & $\begin{array}{l}\text { Evolutionary equilibrium (continuously stable } \\
\text { strategy, CSS) }\end{array}$ \\
\hline & Subscripts \\
\hline$c$ & $\begin{array}{l}\text { Phenotypic constraints (assumed traits) in a } \\
\text { historical selection }\end{array}$ \\
\hline$e$ & $\begin{array}{l}\text { Phenotypic traits to be explained by a historical } \\
\text { selection }\end{array}$ \\
\hline
\end{tabular}

